\title{
A State-of-the-Art Review of the Sensor Location, Flow Observability, Estimation, and Prediction Problems in Traffic Networks
}

\author{
Enrique Castillo, ${ }^{1}$ Zacarías Grande, ${ }^{1}$ Aida Calviño, ${ }^{2}$ W. Y. Szeto, ${ }^{3}$ and Hong K. Lo ${ }^{4}$ \\ ${ }^{1}$ Department of Applied Mathematics and Computational Sciences, University of Cantabria, 39005 Santander, Spain \\ ${ }^{2}$ Department of Computer Science and Mathematics, University of Rovira i Virgili, 43007 Tarragona, Spain \\ ${ }^{3}$ Department of Civil Engineering, The University of Hong Kong, 999077 Pok Fu Lam, Hong Kong \\ ${ }^{4}$ Department of Civil and Environmental Engineering, Hong Kong University of Science and Technology, \\ 999077 Sai Kung District, Hong Kong
}

Correspondence should be addressed to Zacarías Grande; zgandrade@gmail.com

Received 1 June 2015; Revised 17 September 2015; Accepted 20 September 2015

Academic Editor: Fanli Meng

Copyright (C) 2015 Enrique Castillo et al. This is an open access article distributed under the Creative Commons Attribution License, which permits unrestricted use, distribution, and reproduction in any medium, provided the original work is properly cited.

\begin{abstract}
A state-of-the-art review of flow observability, estimation, and prediction problems in traffic networks is performed. Since mathematical optimization provides a general framework for all of them, an integrated approach is used to perform the analysis of these problems and consider them as different optimization problems whose data, variables, constraints, and objective functions are the main elements that characterize the problems proposed by different authors. For example, counted, scanned or "a priori" data are the most common data sources; conservation laws, flow nonnegativity, link capacity, flow definition, observation, flow propagation, and specific model requirements form the most common constraints; and least squares, likelihood, possible relative error, mean absolute relative error, and so forth constitute the bases for the objective functions or metrics. The high number of possible combinations of these elements justifies the existence of a wide collection of methods for analyzing static and dynamic situations.
\end{abstract}

\section{Introduction}

The problem of knowing how traffic behaves in areas of different zones (cities, regions, etc.) has deserved the attention of researchers for a long period, not only because of its theoretical interest but because of its important practical implications. Observing, estimating, and predicting link, OD, and route flows are considered as very relevant problems in the traffic field, so that many papers have dealt with this problem. In this context, the role of sensors and their location and the flow observability, estimation, and prediction problems become important. Though sensors can be used for many different purposes in the traffic field (see, e.g., Gil Jiménez and Fernández-Getino García [1], Liu et al. [2], and Kong et al. [3]), in this paper, we use sensors to determine the traffic flow. In particular, it is important to distinguish between passive and active sensors. Following Gentili and Mirchandani [4], we call passive sensors to those sensors that simply count vehicles at points in a network and active sensors to those able to identify vehicles, so that routes can be determined. For a detailed description of the different types of sensors see Gentili and Mirchandani [4]. The difference between active and passive sensors is very relevant to the studied problem because the first provide much more information on flows and in particular on route flows, not provided by the last ones. Typical questions of location problems arising in a natural form are the following: determining how many, what type, and where they must be located to observe a given set of flows.

In this paper, we review the existing literature, aiming at trying to analyze the different approaches. However, before starting our study, we want to mention and recognize some previous works related to the reviews of existing works that can be useful to readers. For example, Branston [5] reviews the state of the art of link capacity functions, or volumedelay curves, for use in traffic assignment procedures; Abrahamsson [6] presents a survey of generic approaches and an 
annotated bibliography of some individual contributions on the OD matrix estimation problem based on traffic counts on links in the transport network and other available information; Montoya-Zamora et al. [7] compare different methods and algorithms; Li et al. [8] present an overview of dynamic traffic assignment models using the variational inequality method and describe the equivalent relations between the variational inequality model and the dynamic user optimal condition model; Peeta and Ziliaskopoulos [9] provide an important state-of-the-art review of dynamic traffic assignment models; Boyce et al. [10] review some analytical formulations of the dynamic traffic assignment problem under a variational inequality point of view; and Szeto and Lo [11] present a very complete and excellent analysis of the dynamic traffic assignment problem and suggest new lines of research.

In this work, we distinguish between observability, estimation, and prediction problems. In the first, we look for information derived exclusively from sensors and other general mathematical relations that need to be satisfied, such as conservation or balance laws, flow nonnegativeness relations, or different flow definition relations. In the second, we add to the previous sources of knowledge about the network other information such as prior flow information and/or statistical models that cover the lack of knowledge required for a proper estimation. Finally, in the third one, we use models to simulate travelers' route choice behavior and load traffic according to the network for prediction purposes.

(1) The Flow Observability Problem. Given a traffic network $(\mathcal{N}, \mathscr{L})$, where $\mathscr{N}$ and $\mathscr{L}$ are the sets of nodes and links, respectively, and assuming that a given subset $\mathcal{O}$ (observed links) of flows have been determined by installing a set of traffic measuring devices, a subset of unobserved flows $\mathcal{U}$ is said to be observable if its flows can be calculated in terms of the observed flows in $\mathcal{O}$. We note that in general the set $\mathcal{O}$ can include any type of flows, such as link, OD, and route flows.

In other words, given the target subset $\mathcal{U}$ of flows, the typical observability problem consists of determining which minimum set of flows $\mathcal{O}$ must be observed (measured) in order to be able to calculate the flows in $\mathcal{U}$.

The reason why the flows in $\mathcal{U}$ can be calculated based on the flows in $\mathcal{O}$ is that all these flows are not independent but related. Thus, the knowledge of all the relations among flows is crucial in the observability problem.

It is important to realize that in the observability problem we look for exact flows. This differentiates this problem from the other two, estimation and prediction of flows, where only some estimates (approximate but not exact flows) are the aim of the analyses.

The above observability problem, defined for the static case, can be extended to the dynamic case and then flows must be understood as flow-time functions. In other words, in the dynamic case we are interested in observing how flows change with time. For the dynamic case, the time delay in arrival from upstream links to downstream nodes needs to be incorporated for maintaining the conservation conditions. We must note, however, that almost all works related to the observability problem deal with the static case. Thus, some research for the dynamic case in this direction would be very useful.

For the sake of illustration, we include below a simple observability problem for the static case, consisting of observing a given subset of routes with a minimum number of cameras (active sensors) and determining where to locate them. The problem written as an optimization problem is (see Castillo et al. [12])

$$
M_{1}=\min _{\mathbf{y}, \mathbf{z}, n_{c}} n_{c}-\rho_{1} \sum_{r \in \mathscr{R}} y_{r}
$$

subject to

$$
\begin{aligned}
& \sum_{a \in \mathscr{A} \mid \delta_{a}^{r}+\delta_{a}^{r_{1}}=1} z_{a} \geq y_{r} ; \quad \forall r, r_{1} \in R ; \quad r \neq r_{1} \in \mathscr{R}, \\
& \sum_{a \in \mathscr{A}} z_{a} \delta_{a}^{r} \geq y_{r} ; \quad \forall r \in \mathscr{R} \\
& \sum_{a \in \mathscr{A}} z_{a}=n_{c}, \\
& y_{r}=1, \quad \forall r \in \mathscr{R}_{\mathrm{obs}} \\
& n_{c} \leq n_{c}^{\max }, \\
& \sum_{a \in \mathscr{A}} z_{a} \delta_{a}^{r} \leq n_{\max } ; \quad \forall r \in \mathscr{R}
\end{aligned}
$$

where $n_{c}$ is the minimum number of required cameras; $z_{a}$ is a binary variable that equals one if link $a$ contains a sensor and zero; otherwise $y_{r}$ is a binary variable that equals one if route $r$ is observed (can be distinguished from others) and zero; otherwise, $\mathbf{y}$ and $\mathbf{z}$ are the vectors of the corresponding variables; $\mathscr{R}$ is the set of routes; $\mathscr{A}$ is the set of links; $\delta_{a}^{r}$ is the element in ath row and $r$ th column of the link-route incidence matrix; $\mathscr{R}_{\text {obs }}$ is the subset of routes that we want to observe; $n_{c}^{\max }$ is the maximum number of available cameras; $n_{\max }$ is the maximum number of scanned links per route; and $\rho_{1}$ is a nonnegative small number.

Objective function (1) minimizes the number of cameras and among all solutions chooses the one with the most observed routes. Constraint (2), if the binary variable $y_{r}$ is equal to 1 , guarantees that route $r$ is able to be distinguished by the subset of scanned links from the other routes. Note that if $\delta_{a}^{r}+\delta_{a}^{r_{1}}=1$, link $a$ belongs only to one of the routes and that if $\sum z_{a} \geq y_{r}$ and $y_{r}=1$, at least one scanned link has this property. On the other hand, if $y_{r}=0$, the constraint always holds. Constraint (3) if $y_{r}=1$ ensures that route $r$, which is able to be distinguished by the subset of scanned links from other routes, contains at least one scanned link. Constraint (4) guarantees that $n_{c}$ cameras are used. Constraint (5) guarantees that each route in $\mathscr{R}_{\text {obs }}$ is identified by the subset of scanned links. Constraint (6) is a budget constraint, which limits the number of cameras to a maximum $n_{c}^{\max }$. Finally, constraint (7) limits the maximum number of scanned links in any route to $n_{\max }$. 
The interested reader can see other simple observability problems for the static case in Yang and Zhou [13] or Gentili and Mirchandani [14].

(2) The Flow Estimation Problem. If flows are unobservable or there are variations in the observations, the observability methods are not sufficient and we must look for alternative options; then the estimation problem arises. The problem of estimating a set of flows consists of using methods to guess the unobservable flows in this set with maximum possible precision. To this end, observed flows and other sources of information, such as flow prior information or mathematical models and statistical methods, must be used. The estimation problem refers to past flow values, that is, flows that could have been measured but were not. Typical examples of traffic estimation problems are determining point estimates or confidence intervals of link, OD, or route flows.

A simple example of the flow estimation problem for the static case stated as an optimization problem is the following:

$$
\begin{aligned}
\min _{\mathbf{f}, \mathbf{t}, \mathbf{v}} Z= & \rho_{1} \sum_{r, e}\left(\frac{f_{r}-f_{r}^{0}}{f_{r}^{0}}\right) \omega_{r, e}^{f}\left(\frac{f_{e}-f_{e}^{0}}{f_{e}^{0}}\right) \\
& +\rho_{2} \sum_{k, e}\left(\frac{\tau_{k}-\tau_{k}^{0}}{\tau_{k}^{0}}\right) \omega_{k, e}^{\tau}\left(\frac{\tau_{e}-\tau_{e}^{0}}{\tau_{e}^{0}}\right) \\
& +\sum_{a, e}\left(\frac{v_{a}-v_{a}^{0}}{v_{a}^{0}}\right) \omega_{a, e}^{\ell}\left(\frac{v_{e}-v_{e}^{0}}{v_{e}^{0}}\right)
\end{aligned}
$$

subject to the constraints

$$
\begin{aligned}
& \tau_{k}=\sum_{r \in \mathscr{R}_{w}} f_{r} ; \quad k \in \mathscr{W}, \\
& v_{a}=\sum_{r \in \mathscr{R}} \delta_{r}^{a} f_{r} ; \quad a \in \mathscr{L}, \\
& \widehat{v}_{a}=v_{a} ; \quad a \in \mathscr{C}, \\
& \widehat{w}_{s}=\sum_{r \in \mathscr{R}} f_{r} \eta_{r}^{s} ; \quad s \in \mathcal{S}, \\
& f_{r} \geq 0 ; \quad r \in \mathscr{R},
\end{aligned}
$$

where $\mathbf{f}, \mathbf{t}$, and $\mathbf{v}$ are the vectors of route, OD, and link flows, respectively, $\rho_{1}$ and $\rho_{2}$ are nonnegative numbers that measure the relative weight we give to the prior information of route and OD flows with respect to link flows, $f_{e}, \tau_{e}$, and $v_{e}$ and $f_{e}^{0}$, $\tau_{e}^{0}$, and $v_{e}^{0}$ are the route, OD, and link flows and the corresponding previous values, respectively, $\boldsymbol{\omega}_{r, e}^{f}, \boldsymbol{\omega}_{k, e}^{\tau}$, and $\boldsymbol{\omega}_{a, e}^{\ell}$ are the elements of the covariance matrices associated with route, $\mathrm{OD}$, and link flows, respectively, $v_{a}$ is the flow on link $a, \widehat{v}_{a}$ are the counted (observed) flows on link $a, \mathscr{C}$ is the subset of links where the flow is counted, $\mathcal{S}$ is the subset of all observed combinations of links associated with the different cameras, $\eta_{r}^{s}$ is the element of route-scanned combination incidence matrix for route $r$, which equals one if route $r$ contains the subset $s \subseteq \mathcal{S}$ of scanned links and no more scanned links and zero otherwise, and $\widehat{w}_{s}$ is the flow associated with the plate scanned link combination $s$.

Objective function (8) is a least squares function, constraints (9) and (10) are the relations between OD and link flows with route flows, respectively, constraints (11) and (12) force the corresponding flows to coincide with the observed flows, and (13) forces the nonnegativity of route flows.

Solving this problem, estimates of all flows $f_{r}, v_{a}$, and $\tau_{k}$ can be obtained. Note that since the observed flows are not sufficient to estimate all flows, we use extra information, normally previously observed flows, and a least squares metric to get the estimated flows. The interested reader can see other simple estimation problems in Cascetta [15] or Papola and Marzano [16].

Since observations play a key role in this type of problems, the optimization problem above can be complicated by including some location problems and then we obtain a special case of problem known as "sensor location flow estimation problem" (see Gentili and Mirchandani [14]). The problems analyzed in this paper are very general and include these as particular cases.

In addition, the flows can be replaced by flow-time functions and then we have dynamic estimation problems, which are stochastic estimation problems. Research in this direction is needed too.

(3) The Prediction Problems. The flow prediction problem consists in predicting a certain subset of flows that will take place under given circumstances. Prediction models are mainly based on statistical traffic data and on simulating the route choice behaviors of users or drivers, assuming either user equilibrium or system optimal or stochastic user equilibrium. In the first case, we have macro, meso, and micro models and also static and dynamic models, point queue, or spatial queue models, depending on the detail at which the traffic is modelled. Prediction models use mathematical equations to reproduce reality and previous data on which the predictions or the model parameters are based on. Typical examples of prediction problems involve questions such as the following: how do the traffic flows change if some changes in the traffic network are done?

Contrary to the estimation problem, the prediction problem refers to future flow values; that is, only flows that occurred at the prediction time can be measured.

In prediction problems the mathematical models reproducing the vehicle behavior play a relevant role and constitute its main components. However, in some cases, estimation models are complemented with these mathematical models and then they can be considered in the border between estimation and prediction models.

A very simple example of the flow prediction problem stated as an optimization problem, often referred to as the Beckmann transformation, is the following:

$$
\min _{\mathbf{f}, \mathbf{v}} Z=\sum_{a \in \mathscr{A}} \int_{0}^{v_{a}} c_{a}(v) d v=\sum_{a \in \mathscr{A}} C_{a}\left(v_{a}\right)
$$


subject to the constraints

$$
\begin{aligned}
& \sum_{r \in \mathscr{R}} \xi_{r w} f_{r}=\tau_{w} ; \quad w \in \mathscr{O} \mathscr{D}, \\
& \sum_{r \in \mathscr{R}} f_{r} \delta_{a}^{r}=v_{a} \quad a \in \mathscr{A}, \\
& f_{r} \geq 0 ; \quad r \in \mathscr{R},
\end{aligned}
$$

where $\mathbf{f}$ and $\mathbf{v}$ are the vectors of route and link flows, respectively, $v$ is a dummy variable, $c_{a}\left(v_{a}\right)$ is the link $a$ travel time function (e.g., the BPR function), $C_{a}\left(v_{a}\right)$ is its integral, $\xi_{r w}$ is the element of the route-OD incidence matrix, which equals 1 if route $r$ belongs to OD pair $w$ and zero otherwise, and $\delta_{a}^{r}$ is one if link $a$ belongs to route $r$ and zero otherwise. Note that the travel time function $c_{a}\left(v_{a}\right)$ reproduces the congestion effect. Note also that the selection of the objective function in (14) implies a Wardrop solution; that is, vehicles with the same OD use only some routes that have the same associated minimum travel time.

This problem can be complicated by adding flow observation and sensor location constraints and variables and considering the dynamic case, that is, time-dependent flow travel time functions, rather than static travel time functions. Thus, in this paper we understand the prediction problem in a general sense.

\section{An Integrated View of the Problems}

With a great generality, we can state all the above as optimization problems. In fact, mathematical optimization provides a general framework to all of them. In order to understand an integrated view of all the previous problems, we dedicate this section to analyze the different components involved in traffic flow problems when considered as optimization problems.

As it becomes apparent from the previous three examples, an optimization problem has four main components as follows.

(1) Data. They mainly consist of the network topology and the observed flows but in some cases can include flow prior information and some flow parameters such as OD-link proportions, route-OD, or node-link probability choices.

(2) Variables. They are the unknown flows, the number of sensors, and their locations and can include some of the above parameters: OD-link proportions, route-OD or node-link probability choices, and so forth, when they are unknown.

The optimal variable values resulting from solving the optimization problem provide the solution to the problem being considered.

(3) Constraints. They are relations that must be satisfied by the unknowns for them to provide a credible and realistic solution.

There are several types of constraints:

(a) Conservation constraints: they express a flow balance that always must be satisfied. For example, if no flow is retained at the nodes, the total flow entering any node must be equal to the total flow exiting the node.

(b) Nonnegativity of flows: it is obvious that unidirectional flows cannot be negative.

(c) Bounded link capacities: these are a clear physical condition.

(d) Flow definition constraints: they are a consequence of the definition of certain flows such as link, OD, and route flows and their relations.

(e) Choice probability definition: this is a consequence of the definition of choice probabilities.

(f) Observation constraints: when flow data are observed, we can enforce the network flows to be consistent with the observations. These relations take a different form depending on the type of data (counts, plate scanned data, AVI, etc.).

(g) Flow propagation constraints: they depict how flow propagates within a link and reproduce how congestion increases the link travel time.

(h) Specific model constraints: if some models are used to improve the knowledge of the traffic behaviour, they provide relations that increase the number of constraints to be satisfied.

We note that the first six types of constraints are linear, but the rest can be nonlinear.

The model constraints play a very important role in observability, estimation, and prediction problems. In particular, if the system of constraints has a unique solution, the optimization problem degenerates and we can reduce our problem for solving the system of constraints to get the desired flow solution and/or sensor locations. This can occur, for example, when plate scanning data is sufficient to identify uniquely all flows involved in the problem.

However, the set of constraints normally has infinitely many solutions and we need to add more constraints if a unique solution is desired. The most common methods to add this complementary information are the least squares and the generalized least squares methods. For example, some authors minimize the sum of squares of the differences between the predicted and the prior matrices, correcting for the variances and covariances of each flow, as Cascetta [17], who uses the link traffic counts to estimate the OD matrix by means of an assignment model, proposes a generalized least squares estimator, and considers the measurement errors and the temporal variability in the observed flows (see also Cascetta and Nguyen [18], Doblas and Benítez [19], Wong et al. [20], Castillo et al. [21], and Mínguez et al. [22]).

It is also important to realize that the above constraints are not necessarily independent; that is, there can be some redundancies that must be taken into account. It is important to realize that redundancy can lead to incompatibility of the system of constraints in which case the resulting optimization problem has no solution. Incompatibility is real in practice when we have redundant constraints and/or observe redundant data because measurements have errors that can lead to inconsistency. In this case, the corresponding equality 
constraints must be replaced, for example, by least squares type constraints, which allow us to consider these errors and minimize them. For example, Li and Ouyang [23] propose a reliable facility location model to optimize traffic travel time estimation in addition to individual sensor flow coverage, while considering probabilistic sensor failures.

(4) Objective Function. This element is crucial in an optimization problem and expresses the criterion under which we select one optimal solution among all the consistent candidate solutions.

We note that when the system of constraints is consistent but undetermined, the optimality conditions (Karush, Kuhn, and Tucker (KKT) or other ones) complete and replace the required set of constraints to limit the set of feasible solutions and lead to a unique solution in many cases. Under this point of view the optimality conditions can be considered as an additional set of constraints that we finally impose to our problem.

In this paper we use as reference the above structure to discuss the different approaches used to solve the flow observability, estimation, and prediction problems.

Before analyzing the existing solutions given to the observability problem in the existing literature, it is convenient to discuss in detail the different elements: data, constraints, and objective functions that appear in traffic problems. This is the aim of the following sections.

2.1. Data Components. As previously indicated, data consists of several components. Probably, the most important data is the network topology that reveals and limits the possible vehicle displacements. If some models are used, the model parameters can also be a part of the data. Though difficult to obtain, sometimes this information is complemented with the allowable routes. In addition, the observed data obtained from active or passive sensors are basic to solve these problems. However, when observed data are not sufficient for observability, they can be complemented with prior information to allow for unique estimates. This supplementary information consists of prior or obsolete data $f_{r}^{0}, \tau_{k}^{0}$, and $v_{a}^{0}$ of route, OD, and/or link flows, respectively.

In the case of underspecification, the only possibility for solving the problem and getting a unique solution consists of adding some extra information, which normally comes in the form of a combination of prior information and observed flows together with some optimization property.

2.2. Constraints. For the sake of generality and to facilitate the understanding, we first present the dynamic case and later we deal with the static case (a.k.a. steady state, fixed point, etc.). See Friesz et al. [24], Cantarella and Cascetta [25], and Lo and Szeto [26] for a particular example.

2.2.1. Traffic Flow Dynamics. We start by saying that obviously the traffic flow dynamics case is much more complicated than the static one (see Cascetta et al. [27]).

In dynamic traffic assignment we normally consider two components: travel choice and traffic flow. At first we analyze how the users select their routes. Second we analyze how traffic propagates in a network once the users have decided where to travel and which routes are used.

In this context, we have the following dynamic constraints.

(1) Dynamic Conservation Equations. They correspond to the flow conservation laws at the nodes. The conservation equations (total flow entering the node $=$ total flow exiting the node) for the link flows are

$$
\begin{aligned}
\sum_{a \in \mathscr{I}_{n}} V_{a}^{i}(t)-\sum_{a \in \mathscr{O}_{n}} V_{a}^{o}(t)=I_{n}(t)-O_{n}(t) & \\
& n \in \mathcal{N} ; t \in \mathscr{T},
\end{aligned}
$$

where $V_{a}^{i}(t)$ and $V_{a}^{o}(t)$ are the cumulated flow that have entered and left link $a$ at time $t$, respectively, $\mathscr{I}_{n}$ and $\mathcal{O}_{n}$ are the set of all links that supply or take flow from the node $n$, respectively, $I_{n}(t)$ and $O_{n}(t)$ are the cumulated external flows entering and exiting node $n$ up to time $t$, respectively, and $\mathscr{T}$ is the set of selected times:

$$
V_{a}^{i}(t)=V_{a}^{o}\left(t+d_{a}(t)\right)
$$

and $d_{a}(t)$ is the travel time of a vehicle that enters link $a$ at time $t$.

The set of (16) implies one conservation equation per node $n$ (finite number) and time $t$ in set $\mathscr{T}$ (finite or infinite). The main advantage of considering cumulated flows $V_{a}^{i}(t)$ instead of flow intensities is that we do not need to use differential equations but functional equations. In other words, this statement avoids derivatives. Replacing differential equations by functional equations is very relevant and should not be overlooked (see Wu et al. [28] and Castillo et al. [29]).

(2) Nonnegativity of Link Flows. Since flows cannot be negative, we must have an increasing cumulative flow function and

$$
V_{a}^{i}(0) \geq 0 ; \quad a \in \mathscr{L} .
$$

If this condition is not explicitly considered, we can have invalid (negative) flow solutions.

(3) Link Capacity Constraints. Since the link capacities are finite, we must have bounded link flows; that is,

$$
V_{a}^{i}(t)-V_{a}^{i}(t-\varepsilon) \leq \varepsilon c p_{a} ; \quad a \in \mathscr{L} ; t \in \mathscr{T},
$$

where $c p_{a}$ is the capacity of link $a$ and $\varepsilon$ is an arbitrary small positive number.

This is another important condition that should not be forgotten. For example, Bierlaire [30] mentions that unbounded flows can arise in some models. This is the consequence of omitting this constraint.

(4) Flow Definition Constraints. The definitions of OD and route flows lead to the following set of constraints. 


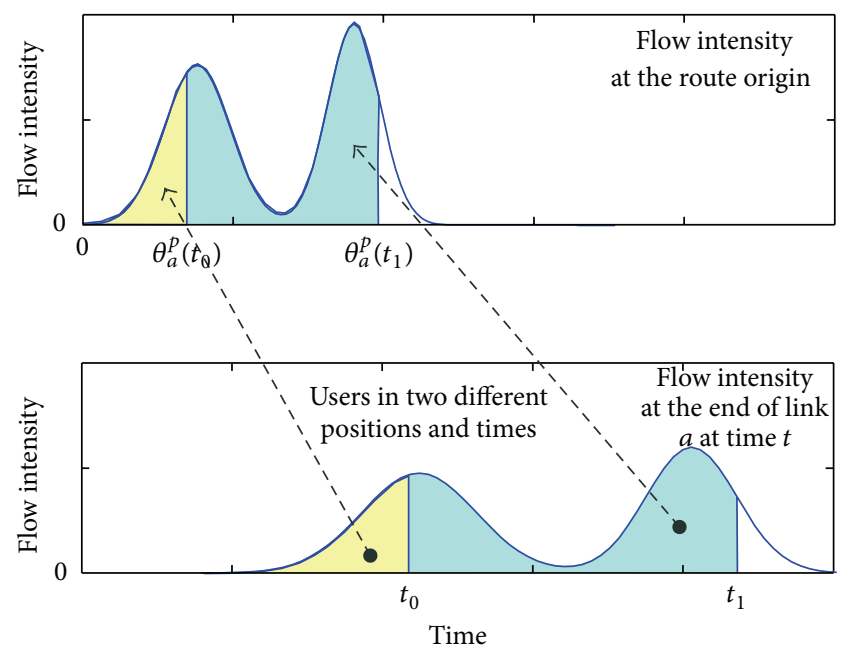

Figure 1: Illustration of how the traffic profiles associated with different points and times are related.

(i) OD-Link Flow Relations. The OD-link flow relations are given by

$$
\begin{aligned}
& V_{a}^{i}(t)=\sum_{w \in \mathscr{O} \mathscr{D}} \sum_{r \in \mathscr{R}_{w}} \delta_{a}^{r} p_{r w}\left(\theta_{a}^{r}(t)\right) T_{w}\left(\theta_{a}^{r}(t)\right) ; \\
& a \in \mathscr{L} ; t \in \mathscr{T},
\end{aligned}
$$

where $\mathscr{R}_{w}$ is the set of routes of OD pair $w, \delta_{r}^{a}$ is the element of the link-route incidence matrix and equals one if link $a$ is on route $r$ and equals zero otherwise; $\mathscr{W}$ is the set of all OD pairs and $p_{r w}(t)$ is the proportion of cumulated users choosing route $r$ in OD pair $w$ at time $t$ and $T_{w}(t)$ is the cumulated flow that has entered a route in OD pair $w$ at time $t$ and $\theta_{a}^{r}(t)$ is the entry time to route $r$ of a vehicle entering link $a$ at time $t$ (see Figure 1).

(ii) OD-Route Flow Relations. The OD-route flow relations are given by

$$
T_{w}(t)=\sum_{r \in \mathscr{R}_{w}} F_{r}(t) ; \quad t \in \mathscr{T} ; w \in \mathscr{W}
$$

where $F_{r}(t)$ is the cumulated flow that has entered route $r$ at time $t$.

These relations (21) can also be written as

$$
F_{r}(t)=p_{r w}(t) T_{w}(t) \quad t \in \mathscr{T}, r \in \mathscr{R}
$$

where $\mathscr{R}$ is the set of all routes.

(iii) Route-Link Flow Relations. The route-link flow relations are given by

$$
V_{a}^{i}(t)=\sum_{r \in \mathscr{R}} \delta_{r}^{a} F_{r}\left(\theta_{a}^{r}(t)\right) ; \quad a \in \mathscr{L} ; t \in \mathscr{T} .
$$

(5) Observation Constraints. When we observe data, the following relations must hold. (i) Counting Data Information. The most common technique to count link users consists in using link counters that provide information on link flows.

Counting data can be taken into consideration by means of constraints such as

$$
V_{a}^{i}(t)=\widehat{V}_{a}^{i}(t) ; \quad a \in \mathscr{C}, t \in \mathscr{T}^{*}
$$

where $\widehat{V}_{a}^{i}(t)$ is the counted cumulated flow that has entered link $a$ at time $t, \mathscr{C}$ is the subset of links where the flow is counted, and $\mathscr{T}^{*}$ is the set of times at which data are collected.

(ii) Plate Scanning Data Information. If a set of cameras are located to register car plate numbers of vehicles travelling on links, plate scanning data can also be considered by means of constraints:

$$
\widehat{W}_{s}(t)=\sum_{s \in \mathcal{S}} \eta_{r}^{s} F_{r}\left(\theta_{a}^{r}(t)\right) ; \quad s \in \mathcal{S}, t \in \mathscr{T}^{*},
$$

where $\delta$ is the subset of all observed combinations of links associated with cameras, $\widehat{W}_{s}(t)$ is the total plate scanned observations in subset $s$ at time $t$, and $\eta_{r}^{s}$ equals one if route $r$ contains the subset $s$ of scanned links $s \subseteq \mathcal{S}$ and no more scanned links and zero otherwise.

(6) Flow Propagation Constraints. They describe how flow propagate within a link and how link travel time depends on congestion. There are several alternative approaches to formulate the constraints.

(i) Link-based travel time functions: they express the link travel time as a function of the link volume. Some examples are the temporal extensions of the BPR function:

$$
\tau_{a}(t)=\tau_{0 a}\left(1+\kappa\left(\frac{x_{a}(t)}{x_{a}^{\max }}\right)^{\alpha}\right),
$$

where $x_{a}(t)$ is the traffic volume on link $a$ at time $t, \tau_{0 a}$ is the free-flow travel time on link $a$, and $\tau_{a}(t)$ is the link $a$ travel time of a vehicle that enters the link at time $t$.

Some authors as, for example, Boyce et al. [10], propose a mixed function of the form

$$
\tau_{a}(t)=\tau_{a}\left(x_{a}(t), u_{a}(t), w_{a}(t)\right)
$$

where $u_{a}(t)$ is the rate of flow entering link $a$ at time $t, w_{a}(t)$ is the link exiting traffic density, and $x_{a}(t)$ is the link traffic volume.

Unfortunately, these models suffer from realism and consistency (see Merchant and Nemhauser [31] or Astarita [32]).

Some examples are the models used by Friesz et al. [33], Fernández and De Cea [34], and Wu et al. [35].

(ii) FIFO constraints: they guarantee the satisfaction of the FIFO rule (see Astarita [32] or Peeta and Ziliaskopoulos [9]):

$$
w_{a}(t+\tau(t))=\frac{u_{a}(t)}{1+\tau_{a}^{\prime}(t)}
$$


(iii) Exit flow functions: they were suggested by Merchant and Nemhauser [31] and express the flow density exiting link $a$ at time $t$ as a function of congestion; that is,

$$
w_{a}(t)=g_{a}\left(x_{a}(t)\right) .
$$

(iv) Flow-density relations: Daganzo $[36,37]$ proposed the following piecewise linear relationship between traffic flow, $y$, and density, $k$ :

$$
y=\min \left\{V k, Q, W\left(k_{\text {jam }}-k\right)\right\},
$$

where $k_{\text {jam }}, Q, V$, and $W$ denote, respectively, jam density, inflow capacity (or maximum allowable inflow), free-flow speed, and the speed of the backward shock wave (or the backward propagation speed of disturbances in congested traffic).

(v) Different queue models: vehicles can be considered as simple points (point queue models) or as elements occupying a space in the links (physical queue models) (see Szeto and Lo [38], Lo [39], Rubio-Ardanaz et al. [40], Kuwahara and Akamatsu [41], Lo and Szeto [26, 42], Nogal [43], or Calviño [44]). The above constraints belong to point queue models. One possibility for physical queue models is to divide links into moving and queuing sections (see Castillo et al. [45]).

(7) Specific Model Constraints. If we use some model, such as UE or SUE models to reproduce traffic flows in the network, we must add the corresponding constraints. One example is the case of variational type models for route choices in which the constraints express a variational principle, such as

$$
\begin{aligned}
f_{r}(t)\left(\pi_{r}(t)-\pi_{w(r)}(t)\right)=0 ; & r \in \mathscr{R} ; t \in \mathscr{T}, \\
\pi_{r}(t)-\pi_{w(r)}(t) \geq 0 ; & r \in \mathscr{R} ; t \in \mathscr{T},
\end{aligned}
$$

where $\pi_{r}(t)$ and $\pi_{w(r)}(t)$ are the travel time for travelers entering route $r$ at time $t$ and the OD pair $w$ containing route $r$, respectively.

As indicated, we must be careful in selecting the constraints of the model in order to avoid violations of the FIFO rule and causality that means that the speed and travel time of a vehicle must be affected only by the vehicles ahead. For example, Szeto and Lo [11] show that since

$$
x_{a}\left(t+\tau_{a}(t)\right)=W_{a}\left(t+\tau_{a}(t)\right)-U_{a}(t),
$$

the outflow $g_{a}\left(x_{a}\left(t+\tau_{a}(t)\right)\right)$ depends on the vehicles that entered link $a$ before $t+\tau_{a}(t)$, and then causality is violated.

Another important point to be considered is the possibility of redundancy or contradiction of constraints. This occurs, for example, in some mixed approaches that force exit and travel time function constraints that are contradictory.

2.2.2. Static or Steady State Case. The steady state case of the preceding case leads to the well-known system of equations for the static situation.

(1) Conservation Equations. They correspond to the flow conservation at the nodes. In this case the conservation equations (total flow entering the node $=$ total flow exiting the node) for the link flows are

$$
\mathbf{B v}=\sum_{a \in \mathscr{I}_{n}} v_{a}-\sum_{a \in \mathscr{O}_{n}} v_{a}=I_{n}-O_{n} ; \quad n \in \mathcal{N},
$$

where $\mathbf{B}$ is the node-link incidence matrix that contains zeroes, ones, and minus ones, $v_{a}$ is the flow of link $a$, and $I_{n}$ and $O_{n}$ are the external flows entering and exiting node $n$, respectively.

The set of (33) implies one conservation equation per node. The rank of the node-link incidence matrix of a network is $n-1$ (see Strang [46]).

(2) Nonnegativity of Link Flows. Since flows cannot be negative, we must have

$$
v_{a} \geq 0 ; \quad a \in \mathscr{L} .
$$

Unfortunately, sometimes this condition is not explicitly considered and this can lead to invalid (negative) flow solutions.

(3) Congestion and Link Capacity Constraints. Since the link capacities are finite, we must have bounded link flows; that is,

$$
v_{a} \leq c p_{a} ; \quad a \in \mathscr{L}
$$

where $c p_{a}$ is the capacity of link $a$. This is another important condition that should not be forgotten.

\section{(4) Flow Definition Constraints}

(i) OD-Link Flow Relations. The OD-link flow relations are given by

$$
v_{a}=\sum_{w \in \mathscr{W}} \sum_{r \in \mathscr{R}_{w}} \delta_{a}^{r} p_{r w} \tau_{w} ; \quad a \in \mathscr{L},
$$

where $\tau_{w}$ is the flow of OD pair $w, \mathscr{W}$ is the set of ODs, and $f_{r}$ is the flow on route $r$.

(ii) OD-Route Flow Relations. The OD-route flow relations are given by

$$
\tau_{w}=\sum_{r \in \mathscr{R}_{w}} f_{r} ; \quad w \in \mathscr{W} .
$$

Since the OD flows $\tau_{w} ; w \in \mathscr{W}$ are nonnegative numbers, (37) shows that the OD flow vector $\mathbf{t}$ is a cone generated by the column vectors of the OD-route incidence matrix $\xi_{r w}$.

These relations can also be written as

$$
f_{r}=p_{r w} \tau_{w} \quad r \in \mathscr{R}
$$

However, this is not convenient because $p_{r w}$ values are difficult or impossible to obtain.

(iii) Route-Link Flow Relations. The route-link flow relations are given by

$$
v_{a}=\sum_{r \in \mathscr{R}} \delta_{r}^{a} f_{r} ; \quad a \in \mathscr{L}
$$


Since the route flows $f_{r} ; r \in \mathscr{R}$ are nonnegative numbers, (39) shows that the link flow vector $\mathbf{v}$ is a cone generated by the column vectors of the link-route incidence matrix.

(5) Observation Constraints. The constraints associated with the observations in this case become as follows.

(i) Counting Data Information. Counting data can be taken into consideration by means of constraints such as

$$
v_{a}=\widehat{v}_{a} ; \quad a \in \mathscr{C},
$$

where $\widehat{v}_{a}$ is the counted (observed) flow on link $a$ and $\mathscr{C}$ is the subset of links where the flow is counted.

(ii) Plate Scanning Data Information. Plate scanning data can also be considered by means of constraints:

$$
\widehat{w}_{s}=\sum_{r \in \mathscr{R}} f_{r} \eta_{r}^{s} ; \quad s \in \mathcal{S}
$$

where $\delta$ is the subset of all observed combinations of links associated with the different cameras and $\widehat{w}_{s}$ is the number of observations in subset $s \in \mathcal{S}$.

Link flow estimates can be obtained as

$$
\widehat{v}_{a}=\sum_{s \in \mathcal{S}} \widehat{w}_{s} \kappa_{s}^{a}=\sum_{s \in \mathcal{S}} \sum_{r \in \mathscr{R}} f_{r} \eta_{r}^{s} \kappa_{s}^{a} ; \quad a \in \mathscr{L},
$$

where $\kappa_{s}^{a}$ is one if link $a$ appears in the link combination $s$ and zero otherwise.

Since the route flows $f_{r} ; r \in \mathscr{R}$ are nonnegative numbers, (41) shows that the set of plate scanned link flow vectors is a cone generated by the column vectors of the link-scanned combination incidence matrix.

(6) Flow Propagation Constraints. Since flows and travel times are dependent on congestion levels, cost or travel time functions exist that express the cost or travel time as a function of the congestion level. One example is the BPR function:

$$
\tau_{a}=\tau_{0 a}\left(1+\kappa\left(\frac{v_{a}}{v_{a}^{\max }}\right)^{\alpha}\right)
$$

where $\tau_{a}$ and $\tau_{0 a}$ are the travel time and free travel time, respectively, of link $a, \kappa$ and $\alpha$ are positive coefficients, and $v_{a}^{\max }$ is the practical capacity of link $a$.

(7) Specific Model Constraints. Some model constraints can be stated as optimization problems. Some examples are the UE and SUE models.

If the analytical solution of these models is known they can be incorporated directly. One example is the case of the logit model for which the probability of using route $r$ in OD pair $w$ becomes

$$
p_{r w}=\frac{\exp \left(-\theta_{w} \psi_{r}\right)}{\sum_{s \in \mathscr{R}_{w}} \exp \left(-\theta_{w} \psi_{s}\right)} ; \quad w \in \mathscr{W}, r \in \mathscr{R}
$$

where $\psi_{r}$ is the travel time on route $r$ and $\theta_{w}$ is a parameter that scales the perceived travel time by OD pair $w$.
Another example is the variational model for route choice:

$$
\begin{aligned}
f_{r}\left(\pi_{r}-\pi_{w(r)}\right)=0 ; & r \in \mathscr{R}, \\
\pi_{r}-\pi_{w(r)} \geq 0 ; & r \in \mathscr{R},
\end{aligned}
$$

where $\pi_{r}$ and $\pi_{w(r)}$ are the travel time on route $r$ and the minimum travel time in OD pair containing route $r$, respectively. The principle states that only routes with the minimum travel time are used for each OD pair.

(8) Budget Limit Constraints. This is a constraint that should not be forgotten when there are budget limits. Some examples can be found in Ehlert et al. [47] and Mínguez et al. [22] who present some optimization problems to locate link counters taking existing sensors into account, budget limitation, and prior OD data.

2.3. Objective Functions (Metrics). In the existing literature there exist statistical measures to quantify the quality of flow estimates. The most common examples are the root mean square error (RMSE) and the mean absolute error (MAE). For example, Sherali et al. [48] present two models, one based on a least squares estimation approach and the other based on a least absolute norm approach, for the estimation of split parameters that prescribe an OD matrix based on dynamic information regarding entering and exiting traffic volumes through an intersection or a small freeway segment.

Very common methods in the traffic area are the RMSE and MAE that measure the closeness between the observed (true) flows and the estimated flows, no matter if they are link, $\mathrm{OD}$, route or, any other flow values.

In the following, some of the most important measures are described.

(1) Generalized Least Squares (GLS). The traffic flows can be obtained by solving optimization problems similar to the one in (8) (see Cascetta [15]) subject to some of the constraints, such as (33) to (41).

Note that we have used relative errors in the objective function, in order to get dimensionless ratios, and prior values have been selected for normalization in order to avoid the presence of zeros in the denominator.

If the system of constraints has a unique solution, then objective function (8) has no role and the prior data do not affect the solution of the optimization problem. Otherwise, the system of constraints has an infinite number of solutions and the optimal value of the problem depends on the prior values $f_{r}^{0}, \tau_{k}^{0}$, and $v_{a}^{0}$. Thus, we can interpret that the prior $f_{r}^{0}, \tau_{k}^{0}$, and $v_{a}^{0}$ complement the information lacking from the observations. Nevertheless, even in this case of an infinite number of solutions some particular flows can be unique and it is important to discover what the subset of these flows is.

Apart from those already mentioned, the least squares metric was used by other many authors, for example, Gentili and Mirchandani [4], Eisenman et al. [49], Castillo et al. [50], and Gentili and Mirchandani [14]. 
Expression (8) after being modified to take into account the sensor location problem becomes (see Mínguez et al. [22])

$$
\begin{aligned}
Z= & \rho_{1} \sum_{r \in \mathscr{R}} \omega_{r}^{f}\left(\frac{f_{r}^{0}-\sum_{r \in \mathscr{R}} f_{r}^{0} y_{r}}{f_{r}^{0}}\right)^{2} \\
& +\rho_{2} \sum_{w \in \mathscr{W}} \omega_{k}^{t}\left(\frac{\tau_{w}^{0}-\sum_{r \in \mathscr{R}_{w}} f_{r}^{0} y_{r}}{\tau_{w}^{0}}\right)^{2} \\
& +\sum_{a \in \mathscr{A}} \omega_{a}^{\ell}\left(\frac{v_{a}^{0}-\sum_{r \in \mathscr{R}} \delta_{a}^{r} f_{r}^{0} y_{r}}{v_{a}^{0}}\right)^{2}
\end{aligned}
$$

where $\tau_{w}^{0}, f_{r}^{0}$, and $v_{a}^{0}$ are the prior OD, route, and link flows, respectively, $R_{w}$ is the set of routes of OD pair $w$, and $y_{r}$ is a binary variable equal to one if route $r$ is identified (observed) uniquely through the scanned links and zero otherwise.

Note that because of $y_{r}$ binary variable the covered flows are considered in (46).

(2) Mean Absolute Relative Error (MARE). This metric was proposed by Mínguez et al. [22]:

$$
\begin{aligned}
\mathrm{MA} & =\frac{1}{|\mathscr{W}|} \sum_{w \in \mathscr{W}}\left|\frac{\tau_{w}^{0}-\sum_{r \in \mathscr{R}_{w}} f_{r}^{0} y_{r}}{\tau_{w}^{0}}\right| \\
& =\frac{1}{|\mathscr{W}|}\left(\sum_{w \in \mathscr{W}} \sum_{r \in \mathscr{R}_{w}} \frac{f_{r}^{0}\left(1-y_{r}\right)}{\tau_{w}^{0}}\right),
\end{aligned}
$$

which implies that minimizing the MARE is equivalent to minimizing the sum of relative route flows of unobserved routes or equivalently maximizing the sum of relative route flows of observed routes.

(3) Possible Relative Error (PRE). Yang et al. [51] were concerned about the quality of the estimated OD matrix and proposed the concept of "Maximum Possible Relative Error" (MPRE) and showed that it can be formulated as a simple quadratic programming problem. This concept was used by Yang and Zhou [13] too.

The MPRE is the optimal value of the optimization problem:

$$
\underset{w \in \mathscr{W}}{\operatorname{Minimize}} \frac{1}{|\mathscr{W}|} \sum_{w \in \mathscr{W}}\left(\frac{\tau_{w}^{*}-\tau_{w}}{\tau_{w}}\right)^{2}
$$

subject to

$$
\sum_{w \in \mathscr{W}} p_{a w}\left(\tau_{w}^{*}-\tau_{w}\right)=0 ; \quad a \in \mathscr{A}
$$

where $\tau_{w}^{*}$ are the true or reference OD values.

Viti et al. [52] present a new method to optimize the position of traffic counts for reliable state estimation and prediction in complex networks by reformulating the MPRE to minimize the error in link traffic states. A simple solution algorithm to the problem was proposed that uses link flow and travel time correlations between links to select, in sequence, the most representative locations in the network.
(4) Total Demand Scale (TDS). It was proposed by Bierlaire [30] and used by Chen et al. [53] and Gan et al. [54].

The TDS measures the intrinsic undeterminate nature of the OD estimation problem and is independent of the OD estimation method. It is based on the route choice proportions, network topology, and traffic counts. It can be calculated by solving two linear programs:

$$
\begin{aligned}
& \phi_{\max }=\operatorname{Max}_{w \in \mathscr{W}} \sum_{w \in \mathscr{W}} \tau_{w}, \\
& \phi_{\min }=\operatorname{Min}_{w \in \mathscr{W}} \sum_{w \in \mathscr{W}} \tau_{w}
\end{aligned}
$$

subject to

$$
\begin{aligned}
& \sum_{w \in \mathscr{W}} \sum_{r \in \mathscr{R}} \tau_{w} p_{r w} \xi_{r w} \delta_{r}^{a}=\widehat{v}_{a} ; \quad a \in \mathcal{O}, \\
& \tau_{w} \geq 0 ; \quad w \in \mathscr{W},
\end{aligned}
$$

where $\phi_{\max }$ and $\phi_{\min }$ are the maximum and minimum OD flows compatible with the constraints, respectively. Once these two problems have been solved, we can calculate the TDS measure as TDS $=\phi_{\max }-\phi_{\min }$. We note that if TDS $=0$ the set of observations has captured the total demand of the network; if TDS > 0 and finite, then the observations are not capable of capturing the total demand of the network; finally, if TDS is infinity, the flow of at least one OD pair cannot be captured by the observed data.

(5) Matrix Rank (MR). It is the rank matrix of the system of equations used to derive the traffic flows. This rank measures the number of linearly independent equations and thus the amount of information. This metric has been used by Gentili [55], Castillo et al. [50,56], $\mathrm{Ng}[57,58]$, or $\mathrm{He}[59]$.

(6) Flow Amount of Information (FAI). An interesting and not easy to solve problem consists of determining how many linearly independent equations are provided by each set of observations and in particular by a given subset $\delta \mathscr{C}$ of $m$ counted or scanned links when the set of routes is fixed and known. This problem led to Castillo et al. [60] to introduce the concept of flow amount of information supplied by a set of scanned observations as the number of linear independent equations it provides.

While a set of $m$ counted link data can provide at most $m$ linearly independent equations with FAI $=m$, the rank of the scanning information matrix $\mathbf{H}_{w}$ associated with (41) is its number of rows. Thus, the "flow amount of information" of a subset of $m$ scanned links is larger than or equal to $m$ but normally much larger than $m$. The FAI can be easily calculated by the nontrivial formula (see Castillo et al. [12, 60]):

$$
\mathrm{FAI}=\sum_{r \mid n_{r}^{\mathcal{S C}} \neq 0} \frac{1}{m-n_{r}^{\mathcal{S C}}}
$$

where $m=|\mathscr{R}|$ is the number of routes and

$$
n_{r_{1}}^{\mathcal{S C}}=\sum_{r_{2} \neq r_{1}} \min \left(\sum_{a \in \mathcal{S} \mathscr{C}}\left(\delta_{a}^{r_{1}}+\delta_{a}^{r_{2}}\right)\left(1-\delta_{a}^{r_{1}} \delta_{a}^{r_{2}}\right), 1\right) .
$$


(7) Likelihood $(\mathrm{LH})$. In statistical methods one of the most powerful methods is the maximum likelihood that generally provides good estimates. Thus, the likelihood is a good objective function to compare the different feasible solutions. Some examples of authors using this objective function are given next.

Spiess [61] proposes an independent Poisson model with unknown means and a maximum likelihood method for estimating an OD matrix from an observed sample matrix, when the volumes on a subset of the links of the network and/or the total productions and attractions of the zones are known.

Nihan and Davis [62] present a maximum likelihoodbased method to estimate intersection turning and through movement probabilities from entering and exiting counts.

Vardi [63] deals with the problem of estimating the node-to-node traffic intensity from repeated measurements of traffic on the links of a network under Poisson assumptions and two types of traffic-routing regimens: deterministic and Markovian route selection models. They discuss maximum likelihood estimation and related approximations.

Parry and Hazelton [64] deal with the problems of estimation of OD matrices based on link count data and based on observed routes taken by each vehicle. They consider an intermediate problem in which link count data is supplemented by routing information for a fraction of vehicles on the network and develop a statistical model for these combined data sources and derive some tractable normal approximations thereof. They examine likelihood-based inference for these normal models under the assumption that the probability of vehicle tracking is known.

(8) Entropy (ET) Based Measures. Van Zuylen and Willumsen [65] propose two models, based on information optimization and entropy maximisation principles, to estimate OD matrices from traffic counts. The models assume that routes are known and use traffic counts to estimate the most likely OD matrix consistent with observed data and prior information about the trip matrix.

Since the entropy measure is proportional to the logarithm of the determinant of the covariance matrix, Zhou and List [66] suggest using this determinant, which is the product of the eigenvalues associated with the covariance matrix of estimates. It can be interpreted as a measure of the volume of a hyperellipsoid for unknown centered flow variables.

(9) Trace of Posterior Estimates (TPE). The trace of a covariance matrix is used to measure the amount of variations in random variables and corresponds to the circumference of the rectangular region that encloses the covariance matrix ellipsoid. It is the sum of the eigenvalues associated with the covariance matrix. Zhou and List [66] have suggested this measure and was used by Simonelli et al. [67].

(10) Flow Covered (FC). The flow covered principle seeks for the solution that maximizes the flow covered. Some authors using this measure are Yim and Lam [68], Chen et al. [53], and Fei and Mahmassani [69].

(11) Unobserved Variability (UV). It was proposed by Viti and Corman [70] and is defined as the sum of the maximum estimation errors on the unobservable flows. The interested reader can see details in Viti et al. [71].

(12) Correlation (CR) Based Measures. Castillo et al. [21] deal with the problem of estimating and updating the OD matrix and link flows from traffic counts and its optimal location. A combination (bilevel) of an OD pair matrix estimation model based on Bayesian networks and a Wardrop-minimumvariance model is used to estimate OD pair and unobserved link flows based on some observations of links and/or OD pair flows. The Bayesian network model is also used to select the optimal number and locations of the link counters based on minimum correlation among link counts.

(13) Number of Sensors (NS). It was used by $\mathrm{Ng}[57,58]$ and Castillo et al. [72, 73].

(14) Synthetic Dispersion Measure (SDM). The synthetic dispersion measure (SDM) was proposed by Simonelli et al. [67] who consider the variability of the OD matrix estimate and is related to the trace of the covariance matrix of the posterior demand estimate conditional upon a set of sensor locations. In the case of a multivariate normal distribution for the prior demand estimate, the proposed SDM does not depend on the specific values of the counted flows, which are unknown in the planning stage, but just on the locations of such sensors.

The idea becomes clear now. We normally have a system of equations (constraints) that has infinitely many solutions and we want to add extra conditions to the system to get a unique solution if possible. The extra conditions are optimality conditions associated with an objective function or metric and set of constraints. Consequently, the objective function when optimized has the role of restricting the set of feasible solutions to optimal solutions. This raises the problem of how to select an adequate metric and points out the need of understanding what the different metrics mean and what are they suitable for.

Once we have defined what we mean by flow observability, estimation, and prediction problems, we have indicated that all of them can be formulated as optimization problems and we have described the types of data, variables, constraints, and objective functions that occur; we dedicate the following three sections to describe the most important proposed models to solve the three problems.

\section{The Observability Problem}

The observability problem has been intensively studied in the existing literature for the static case but needs research for the dynamic case. Because of their substantial differences, we deal with these two cases separately.

3.1. Some Approaches to the Observability Problem in the Static Case. The observability problem has been solved in the past by many different approaches, most of them assuming the static case, to which we refer in this section.

The observability problems can be classified (see Castillo et al. [12]) as follows: (a) link flow observability, (b) OD flow observability, (c) route flow observability, and (d) general case flow observability. 
3.1.1. Link Flow Observability. The link flow observability problem (see Hu et al. [74]) consists of observing link flows based only on link flow observations.

However, depending on whether or not some route knowledge is assumed and the set of relations among link flows is used, there have been different approaches to this problem, such as (a) node based approaches, (b) OD based approaches, and (c) route based approaches. Next, some of them are discussed.

(1) Node Based Approaches. In this approach, only (33) is considered. The idea consists of determining a minimal subset of linearly independent link flows such that a given set of link flows can be calculated as linear combinations of them.

The set of solutions $\mathbf{v}$ of linear system (33) with (40) and/or (41) is an affine subspace; that is, it can be written as

$$
\mathbf{v}=\mathbf{v}_{0}+\mathbf{N} \boldsymbol{\rho},
$$

where $\mathbf{v}_{0}$ is a particular solution vector of (33) (any solution can be used), $\mathbf{N}$ is the null-space matrix associated with the system (33), and $\boldsymbol{\rho}$ is a column matrix of arbitrary real numbers (see Castillo et al. [75] or [76]).

The rows of matrix $\mathbf{N}$ play an important role in the observability problem because if row $a$ is null (all its elements are zero), the corresponding link flow $v_{a}$ is observable, and the number of nonnull elements in each row is the number of link flows that need to be observed to convert the corresponding link in observable. This permits sorting the links considering how far they are from observability.

Consequently, counting data is sufficient to identify link flows even though not all links are counted, because link flows are linearly dependent.

$\mathrm{Ng}$ [57] proposes for the first time a node based approach that has the advantage of avoiding route enumeration and provides an upper bound $\ell-\bar{n}$, where $\ell$ and $\bar{n}$ are the numbers of links and no-centroid nodes, respectively, for the number of links that need to be observed in order to get observability of all links. The networks considered by Ng [57] have only origin, destination, and intermediate nodes, but no node serves as both an origin and a destination at the same time. $\mathrm{Ng}$ [58] generalized the previous method to the case of partial observability. This is the best bound that can be obtained if only (33) and counted link flows are used. This problem is also discussed by Castillo et al. [77], who present alternative formulas for the link flow estimates and discuss in detail the partial observability problem. Since algebraic approaches are computationally intensive, a graphical method based on evaluating the minimum spanning tree was first suggested by Mori and Tsuzuki [78] who presented a new unified method for dealing with the problems of topological observability in power system state estimation.

Spanning trees were already used by He [59] to solve the link flow observability problem without considering routes. The idea consists of building a network with a virtual node and virtual links to reproduce node inflows and outflows and then working with spanning trees. However, a network with all centroid nodes cannot be analyzed using this method.

Castillo et al. [73] show that the minimum number of links to be counted for complete observability is $\ell-n+c$, where $\ell, n$, and $c$ are the number of links, nodes, and centroid nodes, respectively.

As indicated, node based approaches give the best upper bound for the number of links to be counted in order to have full link observability when route information is not available, that is, based on (33).

It is worth mentioning that system (33) with (40) and/or (41) could be incompatible. There are some methods to test the compatibility of a linear system without solving it (see Castillo et al. $[75,79])$ and even ways of solving simultaneously all its subsystems (see Castillo et al. [80]). In particular, the latter allows us evaluating the contribution of each observation to the observability of the given subset of flows.

In addition, constraints (34) and (35) cannot be ignored. If constraints (34) are added to (33), the set of link flow vectors $\mathbf{v}$ consists of a vector (any particular positive solution of (33)) plus a cone.

If constraints (35) are added to (33) and (34), the set of link flow vectors $\mathbf{v}$ becomes a polytope (a linear convex combination of solution flow vectors).

(2) OD Based Approaches. In these approaches, only (36) together with (40) and/or (41) are considered. The idea consists of determining a minimal subset of linearly independent link flows such that all other link flows can be calculated as linear combinations of them and the OD flows $\tau_{w}$ are satisfied. Several cases can be considered here depending on the assumptions.

(1) The Proportions $\rho_{a w}$ of OD Flow $\tau_{w}$ Using Link a Are Known. In this case, the resulting system of equations contains unknown links $v_{a}$ and OD flows $\tau_{w}$. We note that $\rho_{a w}$ values cannot be given arbitrarily, because a set of routes consistent with these values must exist. In addition, the solution set is normally undetermined and then more link counters are needed in order to get a unique solution.

In this case we have

$$
v_{a}=\sum_{w \in \mathscr{W}} \rho_{a w} \tau_{w} ; \quad a \in \mathscr{L} .
$$

(2) The Proportions $\rho_{a w}$ of OD Flow $\tau_{w}$ Using Link a Are Unknown. In this case, the problem becomes nonlinear because of the product $\rho_{a w} \tau_{w}$ appearing in (36), unless the OD flows are known.

In this group, there are some important contributions. Some are given next.

Hodgson [81] deals with observability and flow estimation problems by finding the minimum number of sensors that guarantee full coverage and maximum captured flow with solutions of a minimal information redundancy (noncannibalizing solutions).

Morrison and Martonosi [82] provide a new necessary condition on the sensor location problem. This condition is not sufficient in general, but for a large class of problem instances the condition is sufficient.

Castillo et al. [50] present a detailed analysis of the plate scanning method to estimate OD flows considering the optimal location of cameras, the prior information, and error analysis. 
Castillo et al. [83] deal with the problem of identifying which subset of OD pair and link flows that can be calculated is based on a subset of observed OD pair and link flows and related problems. Two algebraic methods for solving the observability problems are given: a global approach based on null spaces and a step-by-step procedure allowing updating the information once each piece of information (OD pair or link flow) becomes available. Castillo et al. [56] present a modified topological version of the previous existing algebraic method that is much faster, use much less memory, and present no rounding errors or zero test problems but identify fewer observable flows.

(3) Route Based Approaches. In this approach, (39) are used together with (40) and/or (42) and complete or partial route structure information. It is important to clarify that it is not necessary to include (33), (34), and (35) because they are automatically satisfied by nonnegative and finite route flows. Similarly, OD type equations such as (36) and (37) are automatically satisfied too.

Considering $v_{a}$ and $f_{r}$ as unknowns, the set of solutions to the system involving (39), (40), and (42) is an affine space and the associated null space provides information about the observability of any given sets of $v_{a}$ or $f_{r}$, that is, whether or not they are observable and how far they are from observability. In this case, the required extra FAI for observability of any $v_{a}$ or $f_{r}$ coincides with the number of nonnull elements in the corresponding row of the null-space matrix.

$\mathrm{Hu}$ et al. [74] solve the whole link observability problem (observing all link flows) assuming route information and using the concept of "reduced row echelon form" (RREF), a technique based on the well-known Gaussian elimination method, which emphasizes its algebraic character. Castillo et al. $[77,84]$ provide a pivoting technique to solve the same problem for partial and whole observability and extend the method to plate scanned data; this problem has also been treated by Castillo et al. [72, 77, 84] and $\mathrm{Ng}$ [57] and consists of determining which subset of link flows can be calculated in terms of another subset of link flows but now using route information.

If route information is used, the bound given by node based approaches can be improved with savings reaching in some cases 16\%, as demonstrated in Castillo et al. [72]. However, since enumerating all routes is a difficult task, the first method was improved by considering linearly independent route vectors by Castillo et al. [72]. More precisely, they show that only a subset of linearly independent routes is required and provide a method to select linearly independent route vectors. Finally, they present real examples in which more than $84 \%$ of the routes need not be enumerated.

Rinaldi et al. [85] observe that using $k$-shortest routes for each OD pair can result in an interesting geographical spread of the sensors and in favouring links near the centroids and at the major junctions. They also point out that using linearly independent routes may help in finding lower number of sensors guaranteeing full observability than the number indicated analytically by $\mathrm{Ng}$ [57].

Contrary to node information alone, route information remains fundamental, for example, for the following reasons. (a) It can be combined with flow estimation approaches (e.g., for optimizing location for OD estimation); (b) extra information, such as shortest routes, can be used to refine solutions; and (c) it can be extended to the powerful source of information provided by scanned links.

Some important conclusions resulting from the previous discussion are the following:

(1) Counting data is not sufficient to identify OD and route flows, because normally the number of these flow variables is huge compared with link flows and independent of them.

(2) Plate scanning permits following the car routes and identifying OD pairs and routes if they are conveniently located and in a sufficient number. They provide link, node, OD, and route flows.

3.1.2. OD, Route, and General Case of Flow Observability. One of the most common observability problems in the traffic literature is the OD pair observability problem, in which the OD pair flows are estimated in terms of other flows. Since in most cases the number of independent link flows is much smaller than the number of OD pair flows, even in the case in which all link flows are available, the OD observability problem based on counting links becomes underspecified; that is, the OD flow observability problem based on link flows has an infinite number of solutions. Thus, the OD flow observability problem based on counting links has no satisfactory solution in many cases of real practice, and when it has solution the situation is not realistic.

A more difficult observability problem is the route flow observability problem, in which we aim to observe all route flows. Since knowledge of the route flows leads to knowledge of the OD pairs and link flows, through the conservation equations, the observability problem of route flows is considered as the full observability problem. However, it is the most difficult observability problem, because its solution requires the maximum amount of information. Thus, the route flow observability problem presents the same underspecification difficulties as the OD observability problem, but even more exaggerated.

Therefore, similar methods, based on prior information, or more powerful techniques, such as the scanned link technology, can be used. The plate scanning technique opens the door to full OD and route flow observability, in the sense that installation of sufficient number of cameras permits calculating all OD and route flows.

However, a general case of observability problem can be stated, in which we aim to observe a given subset of flows $\mathcal{U}$ (including any type of flows) and we must determine the subset of observed flows $\mathcal{O}$ in order that this is possible.

Castillo et al. [77, 86] deal with the general observability problem in which link, OD, or route flows are involved. In these cases the use of active sensors is required; in particular, plate scanning is a very effective mean of obtaining flow data.

Table 1 gives the number of unknowns and equations or inequations of the systems of equations used in the different observability options and associated ranks. These ranks 
TABLE 1: Number of unknowns and equations or inequations of different systems of equations and associated ranks.

\begin{tabular}{|c|c|c|c|c|c|c|c|}
\hline \multirow{2}{*}{ Equation } & \multirow{2}{*}{ System } & \multicolumn{3}{|c|}{ \# of unknowns } & \multirow{2}{*}{ \# of equations or inequations } & \multicolumn{2}{|c|}{$\operatorname{Rank}(r)$} \\
\hline & & $v_{a}$ & $\tau_{w}$ & $f_{r}$ & & Lower bound & Upper bound \\
\hline & & & & & Equations & & \\
\hline (33) & Conservation equations & $|\mathscr{L}|$ & - & - & $|\mathcal{N}|$ & $|\mathscr{L}|-k_{0}-h$ & $|\mathscr{L}|-k_{0}-h$ \\
\hline$(40)$ & Counting data & $|\mathscr{C}|$ & - & - & $|\mathscr{C}|$ & 1 & $|\mathscr{C}|$ \\
\hline (41) & Plate scanned data & - & - & $|\mathscr{R}|$ & $|S|$ & $|S|$ & $|S|$ \\
\hline (33), (40), (41) & $\begin{array}{l}\text { Conservation, counts, and } \\
\text { plate scanned equations }\end{array}$ & $|\mathscr{L}|$ & - & $|\mathscr{R}|$ & $|\mathcal{N}|+|\mathscr{C}|+|\mathcal{S}|$ & $|\mathscr{L}|-k_{0}-h+1$ & $|\mathscr{L}|-k_{0}-h+|\mathscr{C}|$ \\
\hline$(37)$ & OD-route flow relations & - & $|\mathscr{W}|$ & $|\mathscr{R}|$ & $|\mathscr{W}|$ & $|\mathscr{W}|$ & $|\mathscr{W}|$ \\
\hline (37), (40), (41) & $\begin{array}{l}\text { Conservation, counts, and } \\
\text { plate scanned equations }\end{array}$ & $|\mathscr{L}|$ & $|\mathscr{W}|$ & $|\mathscr{R}|$ & $|\mathscr{W}|+|\mathscr{C}|+|\mathcal{S}|$ & $|\mathscr{W}|+|\mathcal{S}|+1$ & $|\mathscr{W}|+|\mathcal{S}|+|\mathscr{C}|$ \\
\hline (39) & Route-link flow relations & $|\mathscr{L}|$ & - & $|\mathscr{R}|$ & $|\mathscr{L}|$ & $|\mathscr{L}|$ & $|\mathscr{L}|$ \\
\hline (39), (40), (41) & $\begin{array}{l}\text { Conservation, counts, and } \\
\text { plate scanned equations }\end{array}$ & $|\mathscr{L}|$ & - & $|\mathscr{R}|$ & $|\mathscr{L}|+|\mathscr{C}|+|\mathcal{S}|$ & $|\mathscr{L}|$ & $|\mathscr{L}|+|\mathscr{C}|+|\mathcal{S}|$ \\
\hline & & & & & Inequations & & \\
\hline $\begin{array}{l}(34) \\
(35)\end{array}$ & $\begin{array}{l}\text { Nonnegativity of link flows } \\
\text { Link capacity constraints }\end{array}$ & $\begin{array}{l}|\mathscr{L}| \\
|\mathscr{L}|\end{array}$ & - & - & $\begin{array}{l}|\mathscr{L}| \\
|\mathscr{L}|\end{array}$ & $\begin{array}{l}- \\
-\end{array}$ & $\begin{array}{l}- \\
-\end{array}$ \\
\hline
\end{tabular}

measure the amount of information associated with the corresponding systems of equations.

3.2. The Observability Problem in the Dynamic Case. The main aim of observability analysis in the dynamic case is to know the flows in a given network and how they change with time. The observability problem in this case is completely different from the observability problem in the static one, because the dynamic case implies observing flow-time functions instead of flow values. This means that at several locations we have different flow-time functions. Consequently, we need first to define the following: the flow (what), the location (where), and the time (when) to be observed.

While the observability problem in the static case has been widely discussed in the existing literature, as shown in the previous sections, it is very difficult to find specific research for the dynamic case. In this paper we want to point out the interest in working in this direction.

In the knowledge of how the flow evolves with time in a given network, the link exit time $w_{a}(t)$ and travel time functions $\tau_{a}(t)$ play a very relevant role, because knowledge of these functions together with some simplifying assumptions, such as FIFO, allows us to calculate $\theta_{a}^{r}(t)$ functions, that is, the times at which a vehicle entering link $a$ at time $t$ initiated its route $r$, and then the route travel time functions, that is, how the travel times of each route changes with departure times.

We must realize that we can have access to the large number of $\theta_{a}^{r}(t)$ functions using a reduced set of functions $\tau_{a}(t)$ that can be calculated from them, as follows:

$$
\theta_{a}^{r}(t)=\tau_{a_{1}^{r}}^{-1}\left(\tau_{a_{2}^{r}}^{-1}\left(\cdots \tau_{a_{n-1}^{r}}^{-1}\left(\tau_{a_{n_{r}}^{r}}^{-1}(t)\right)\right)\right)
$$

where $\tau_{a}^{-1}(t)$ is the link $a$ entering time of a user who exits that link at time $t$, that is, the inverse of $\tau_{a}(t)$ function, and $a_{1}^{r}, a_{2}^{r}, \ldots, a_{n_{r}}^{r}$ and $n_{r}$ are the links of route $r$ and its number of links, respectively.

This suggests that we can state a wide collection of dynamic observability problems by solving the observability problem of functions $\tau_{a}(t)$ together with traffic volumes at some locations. This interesting problem is suggested for future research.

\section{The Flow Estimation Problem}

As indicated, when we are unable to observe the desired flows by direct observation and using the conservation and flow definition equations we must use additional information and/or models to estimate flows. The additional information tells us about previous experience so that we can use it for the actual situation assuming that the actual situation has not changed or suffered small changes.

4.1. Static Case. To measure the quality of the flow estimates we normally use a measure. Consequently, many of the existing methods can be classified by considering what this metric is. Some examples of the metrics used and a list of some works using them are given below.

Generalized Least Squares Measures. Least squares are very powerful and interesting methods to solve the estimation problems. Some examples are given below.

Lam and Lo [87] are concerned with the reliability of information and optimize it using generalized least squares; Bierlaire and Toint [88] propose an improvement of existing methods of OD matrix estimation by an explicit use of data, obtained from parking surveys, describing the structure of the matrix, and propose using the GLS metrics; Asakura et al. [89] deal with the problem of OD matrix estimation using the observed data with the AVI system and a least squares model. The results of license plate matching in a pair of AVI cameras are the input variables; Gan et al. [54] present some methods for OD matrix estimation and counter location with error bound analysis in an integrated form and use the MPRE, generalized least squares, and total demand scale (TDS) metrics; Marzano et al. [90] deal with the problem of correction of OD matrices using traffic counts and confirm that the main reason for the data insufficiency stands in the fact that the number of equations is lower than the number of unknowns. To solve the problem, they suggest moving to within-day 
dynamic contexts, where a much larger number of equations are generally available and using GLS methods; Mínguez et al. [22] apply least squares but suggest the use of dimensionless variables.

Likelihood Measures. The likelihood function, coming from the statistical field, has a lot of power for estimation purposes together with a powerful theory supporting it. Some authors using the likelihood as metrics in the classical sense are Spiess [61], Nihan and Davis [62], Vardi [63], and Parry and Hazelton [64]; Bell [91] describes a model that under certain circumstances yields the most likely OD matrix which is consistent with measurements of link traffic volumes; Watling and Maher [92] and Watling [93] suggest using active sensors together with maximum likelihood and best matching techniques to estimate OD matrices; Lo and Chan [94] propose a maximum likelihood procedure for the simultaneous estimation of an OD matrix and link choice proportions from OD survey data and traffic counts for congested networks recognizing that link choice proportions in a network change with traffic conditions; Papola and Marzano [16] using the maximum likelihood principle and the GLS methods show through laboratory experiments that the classical correction of the OD matrix from traffic counts is generally unable to provide effective correction of the OD matrix.

Maximum Coverage. Several authors realized the importance of covering with sensors a maximum of flows, so that the maximum coverage rule has been widely contemplated. For example, Church and ReVelle [95] deal with the maximal covering location problem; Fei et al. [96] identify a set of sensor locations that optimize the coverage of OD demand flows of the road network and maximize the information gains through observation data over the network, while minimizing the uncertainties of the estimated OD demand matrix; Larsson et al. [97] compare different methods for allocating link flow sensors with respect to the quality of the estimated OD matrix and compare three allocation methods. However, they conclude that maximizing the coverage of OD pairs seems to be unfavorable for the quality of the estimated OD matrix; Simonelli et al. [67] provide an innovative and theoretically sound methodology for solving the network sensor location problem, explicitly accounting for the variability of the OD matrix estimate.

Synthetic Dispersion Measure. Simonelli et al. [67] propose a specific measure, termed synthetic dispersion measure (SDM), related to the trace of the covariance matrix of the posterior demand estimate conditional upon a set of sensor locations and an algorithm to work with it.

Expected Information Gain. Zhou and List [66] discuss how to locate a limited set of traffic counting stations and automatic vehicle identification (AVI) readers in a network in order to maximize the expected information gain for the OD demand estimation problem subject to a budget constraint. The model takes into account several important error sources, such as the uncertainty in historical information, sensor measurement errors, and those associated with link proportions.
Based on a mean square measure, the authors provide a stochastic optimization procedure and an algorithm to find suboptimal point and point-to-point sensor locations.

Rules for Reduction of Estimate Uncertainties. Since the very beginning, researchers were concerned with finding rules to obtain optimal locations for sensors. The most relevant were Yang and Zhou [13] who addressed the problem of how to determine the optimal number and the locations of sensors in a road network for a given prior OD distribution pattern and derived four rules: OD covering rule, maximal flow fraction rule, maximal flow-intercepting rule, and link independence rule. In addition, the authors presented integer linear programming models and heuristic algorithms to determine the counting links satisfying these rules; Mínguez et al. [22] provided techniques for obtaining the optimal number and location of plate scanning devices for a given prior OD distribution pattern under maximum route identifiability or budget constraints, developed two rules analogous to the counting location problem, and proposed several integer linear programming models fulfilling these rules.

Since mathematical models reproducing traffic flows are relevant to provide extra equations (constraints), some authors have been concerned about the quality of models that reproduce the traffic behaviour. Wang et al. [98] provide a new linear integer programming model for the placement of sensors to maximize the reduction in the uncertainties in route flow estimates. The model assumes that a general underlying traffic loading model, the route choice set from each OD pair, is known and prior route flows and their reliabilities are given.

Similarly, Chen et al. [99] develop strategies in the screenline-based traffic location problem model for selecting additional traffic counts for improving OD trip table estimation using a SUE principle.

Multiobjective Measures. The observability, estimation, and prediction problems are essentially multiobjective. Thus, several authors dedicate work to the problem of how to deal with multiobjective traffic problems. This is the case of Brenninger-Göthe et al. [100] who present some multiobjective programming formulations for estimating OD matrices and show how multiobjective theory can be used in the interpretation of the problem or Fei and Mahmassani [69] who present a multiobjective model, which considers link information gains and OD demand coverage to locate a minimal number of passive point sensors in a roadway network subject to budget limits.

Bayesian Methods. Among statistically based methods to analyze traffic flows, Bayesian methods appear very useful because they allow us to include the expert knowledge by means of prior distributions. In this context, Maher [101] proposes a method based on Bayesian statistical inference that allows complete flexibility in the degree of belief placed on the prior estimate of the OD trip matrix and also in different parts of the prior estimate; Castillo et al. $[102,103]$ propose an extended gamma-shifted model to estimate traffic flows. The reconstruction of the sample flows can be done exactly 
or approximately, depending on the intensity of the plate scanning sampling procedure. To this end, a generalized least squares technique is used together with the conservation and flow definition laws. A Bayesian approach using special conjugate families is proposed that allows us to estimate different traffic flows, such as route, OD pair, scanned link, or counted link flows.

To end this section, we consider the problems of error recovery and some contributions related to some special solving algorithms.

Error Recovery. The incompatibility problems derived from data replications and the distortion produced by measurement errors led some authors to consider the error recovery problem. For example, Lo et al. [104] review the use of statistical modeling in the estimation of OD matrices from traffic counts and discuss statistical models that consider explicitly the presence of measurement and sampling errors in the observed link flows treating the link choice proportions as random variables; Castillo et al. [50, 105] and SánchezCambronero et al. [106] provide models that permit error identification and recovery.

Branch-and-Bound Methods. Some particular cases, such as the cordon-screen lines, have been treated by Yang et al. [107] who dealt with the optimal selection of cordon-screen lines for traffic census study in road networks that can be stated as how to select the optimal locations of a given number of counting stations to separate as many OD pairs as possible and how to determine the minimum number of counting stations and their locations required for separating all OD pairs. The authors provide a solution scheme that combines a shortest route based column generation procedure and a branch-and-bound technique.

Genetic Algorithms. Since the resulting optimization problems are in some cases too complex, some authors suggest the use of genetic algorithms, such as Chootinan et al. [108], who consider the traffic counting location problem for the purpose of OD trip matrix estimation and determine the number and locations of counting stations that yield maximal coverage and minimal resources. A distance-based genetic algorithm is used to solve the proposed biobjective traffic counting location problem by explicitly generating the nondominated solutions.

Similarly, Cipriani et al. [109] deal with two different heuristic approaches for solving the problem of optimal location of traffic count sections. The first method applies deterministic rules on OD flows and OD pair coverage, but the second uses a genetic algorithm.

Finally, as a summary, we include Table 3 with a long list of contributions, together with the different data, variables, constraints, and objective functions used and the illustrative or real examples utilized. Table 2 provides the symbols and notation used in Table 3.

4.2. Dynamic Case. Most of the works in the existing literature deal with the static and ignore the dynamic case, which is a stochastic process estimation problem. Thus, some research
TABLE 2: Table 3 legend.

\begin{tabular}{|c|c|}
\hline \multicolumn{2}{|r|}{ Data } \\
\hline $\mathrm{CP}$ & Choice probability \\
\hline $\mathrm{L}$ & Link data \\
\hline OD & OD data \\
\hline $\mathrm{P}$ & Route data \\
\hline PA & Route knowledge \\
\hline PI & Prior information \\
\hline \multicolumn{2}{|r|}{ Variable } \\
\hline $\mathrm{CP}$ & Choice probability \\
\hline $\mathrm{L}$ & Link variable \\
\hline OD & OD variable \\
\hline $\mathrm{P}$ & Route variable \\
\hline SL & Sensor location \\
\hline \multicolumn{2}{|r|}{ Constraint } \\
\hline $\mathrm{BC}$ & Bounded capacity \\
\hline $\mathrm{CE}$ & Conservation equation \\
\hline $\mathrm{CP}$ & Choice probability \\
\hline FD & Flow definition \\
\hline FM & Flow model constraint \\
\hline NN & Nonnegativity \\
\hline $\mathrm{OC}$ & Observation constraint \\
\hline $\mathrm{OT}$ & Other constraints \\
\hline \multicolumn{2}{|c|}{ Metric (objective function) } \\
\hline$\overline{\mathrm{BM}}$ & Bayesian model \\
\hline CR & Correlation \\
\hline ET & Entropy \\
\hline FAI & Flow amount of information \\
\hline FC & Flow covered \\
\hline GLS & Generalized least squares \\
\hline IN & Information \\
\hline LH & Likelihood \\
\hline MA & Mean absolute relative error (MARE) \\
\hline $\mathrm{MO}$ & Multiobjective function \\
\hline MP & Maximum possible relative error (MPRE) \\
\hline MR & Matrix rank \\
\hline MUV & Maximum observability variable \\
\hline NS & Number of sensors \\
\hline SD & Synthetic dispersion measure \\
\hline TDS & Total demand scale \\
\hline TPE & Trace of posterior estimates \\
\hline UM & Unobserved variability \\
\hline WMV & Wardrop minimum principle \\
\hline \multicolumn{2}{|r|}{ Examples } \\
\hline $\mathrm{CN}$ & Campania region network \\
\hline $\mathrm{CR}$ & Ciudad Real network \\
\hline $\mathrm{CU}$ & Cuenca network \\
\hline $\mathrm{EN}$ & Edwards network \\
\hline FB & Fishbone \\
\hline $\mathrm{FN}$ & Fuorigrotta network \\
\hline GN & Gateshead network \\
\hline $\mathrm{HC}$ & Helena city \\
\hline HS & Hanshin expressway network \\
\hline IN & Irvine network \\
\hline KA & Kowloon area \\
\hline $\mathrm{LN}$ & Leicester network \\
\hline $\mathrm{MN}$ & Maryland chart network \\
\hline ND & Nguyen-Dupuis network \\
\hline NP & 30 -node problem \\
\hline SF & Sioux Falls network \\
\hline $\mathrm{T}$ & Toy \\
\hline TPE & Triangle park \\
\hline RT & Rotterdam \\
\hline
\end{tabular}




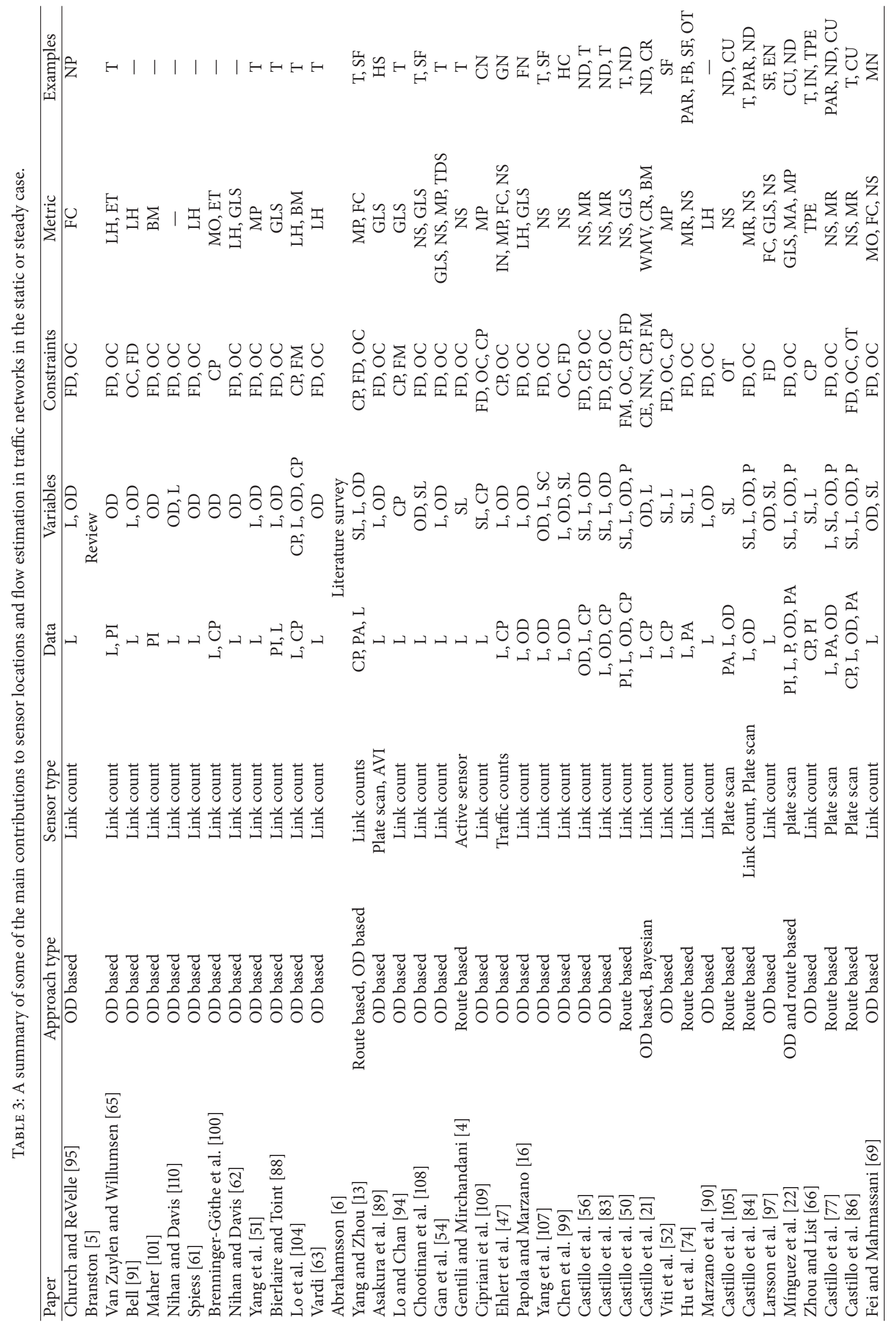




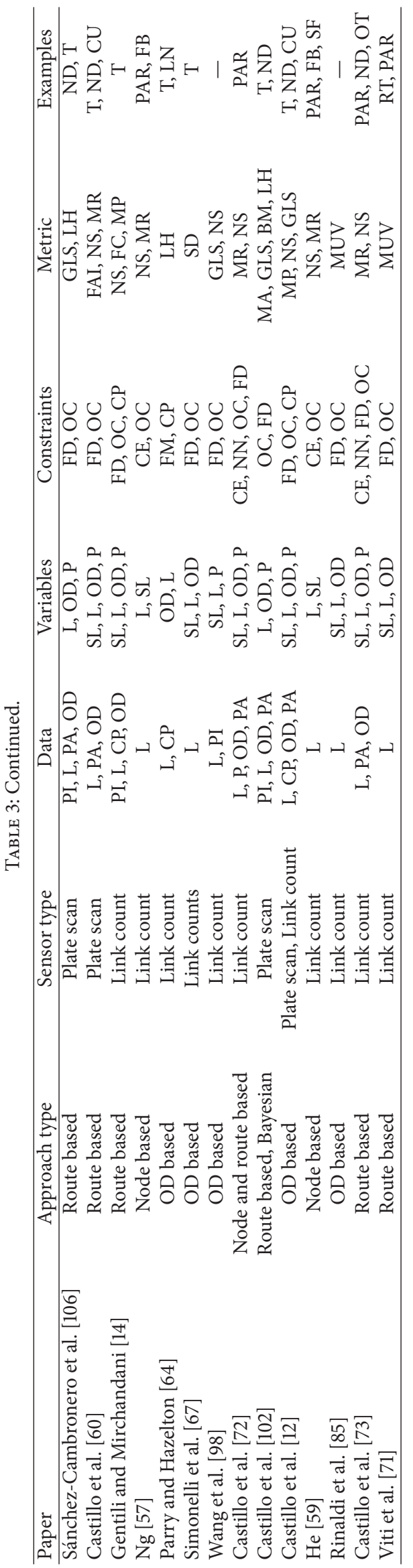


is needed in this direction. One of the few examples of flow estimation in the dynamic case corresponds to the case of time-dependent OD matrix estimation.

Time-Dependent OD Matrix Models. Time-dependent proposals for traffic models were suggested by Ashok and BenAkiva [111] who provide a framework for real-time estimation/prediction of time-dependent OD matrices and apply the model to the Massachusetts Turnpike and Ashok and Ben-Akiva [112] who present models for the estimation and prediction of time-dependent OD matrices. The key contribution of the proposed approach is the explicit modeling and estimation of a stochastic assignment matrix between time-dependent OD flows and link volumes. The assignment matrix is assumed to depend on travel times and route choice fractions in the network; Peterson [113] deals with the problem of estimation of the OD matrices based on observed link counts and considers the time-independent and timedependent cases.

As indicated by Barcelò et al. [114], time-dependent OD matrices are a key input to dynamic traffic models. Microscopic and mesoscopic traffic simulators are relevant examples of such models, which are used in the design of traffic systems. Typical approaches to the time-dependent OD estimation are based either on mathematical programming approaches or on Kalman filter models. However, new data types, such as AVI, plate scanning techniques, and detection of mobile devices, make available traffic data of higher quality allowing for new modeling hypothesis leading to more computationally efficient algorithms. In this context, Barcelò et al. [114] extend the previous research on Kalman filtering approaches for OD estimation to more complex topologies of urban networks, where alternative path choices between origins and destinations are available.

\section{The Flow Prediction Problems}

Models used to solve flow prediction problems incorporate simulation of the traffic behavior; that is, they permit analyzing how changes in the behaviour of the vehicles and infrastructure modify the flows; thus, the models are an important element to be considered.

5.1. Static Models. Some examples of static models for flow predictions are given next.

Boyce and Janson [115] analyze the effect of different link congestion functions on equilibrium assignment; Bell [116] proposes two logit assignment methods that work without route knowledge as alternatives to Dial's algorithm; some models considering multiple class users with overtaking were analyzed in Castillo et al. [117, 118]. Multiclass users with different concerns about travel time are discussed in Castillo et al. [119].

5.2. Dynamic Models. There is a wide collection of dynamic models used for flow prediction. We include below some of them.
Prior Information Based Models. One way to predict flows and flow evolution with time in the dynamic case is storing this information in previous periods and trying to identify the trends from this information. At a given instant, we can know the traffic characteristics at other locations in previous instants, and, looking for similarities or using inference, we can predict future traffic flows. For example, Castillo et al. [29] develop a stochastic demand dynamic traffic model to predict traffic variables (link travel times, flows, densities, etc.) and their time evolution in real networks. The model assumes the variables to be generalized beta variables such that when they are marginally transformed to standard normal all of them become multivariate normal. This gives sufficient degrees of freedom to reproduce (approximate) the considered variables at a discrete set of time-location pairs. The parameters of the model are learned based on previous observations. The model provides point or confidence intervals or the density of the variable being predicted. A closed formula for the conditional future variable values (link travel times or flows), given the available past variable information, is provided. Since only local information is relevant to short-term link flow predictions, the model is applicable to very large networks.

Mathematical Programming Based Traffic Assignment Models. Merchant and Nemhauser [31] consider a dynamic traffic assignment model as a nonlinear and nonconvex mathematical program. Necessary optimality conditions that require marginal costs for all the same OD routes that are being used to be equal are shown to be a generalization of the optimality conditions of the static traffic assignment problem. They also show that the behavior of the dynamic model under static demand conditions is a generalized version of the static model.

Friesz et al. [120] consider two formulations of the dynamic traffic assignment problem, system and user optimizations on a single node network using optimal control theory, and offer the first dynamic generalization of Beckmann's equivalent optimization problem for static user optimized traffic assignment in the form of an optimal control problem.

Jayakrishnan et al. [121] present a dynamic traffic assignment model with traffic flow relationships based on a bilevel optimization framework. Using the modified Greenshields speed-density relationship, they derive a link-cost function that is monotonically nondecreasing and convex with respect to density.

Szeto and Lo [122] compare dynamic traffic assignment models with point and physical queues and discuss their implications together with their solution existence and uniqueness.

Carey and Subrahmanian [123] model link flows considering the flow rate when the vehicle enters and exits the link and knock-on effects from traffic ahead of the link and suggest dividing each link into a travel link followed by a queue link.

Cell Transmission Type Models. The cell transmission model (CTM) is a simple representation of traffic flow that is 
consistent with the kinematic wave theory under all traffic conditions to predict traffic's evolution over time and space, including transient phenomena such as the building, propagation, and dissipation of queues.

One of the first and better known models is Daganzo's cell transmission model (CTM). Daganzo [36, 37, 124] presents the cell transmission model and introduces a numerical procedure for networks, assuming that a time-varying origindestination OD matrix is given and that the proportion of turns at every junction is known or the best routes to each destination from every junction are known at all times. The difference equations are shown to be the discrete analog of the differential equations arising from a special case of the hydrodynamic model of traffic flow reproducing shockwaves in a simple way and showing that the model can mimic the stopand-go traffic within moving queues. Daganzo [125] shows that if the kinematic wave model of freeway traffic flow in its general form is approximated by a particular type of finite difference equation, the finite difference results converge to the kinematic wave solution despite the existence of shocks in the latter. This result is shown not to hold for other commonly used finite difference schemes. An error analysis is also performed.

Lo [39] develops a dynamic traffic assignment formulation, referred to as DUO, based on a dynamic extension of Wardrop's principle. Traffic is modeled as a cell transmission model (CTM), which provides a convergent approximation to the Lighthill and Whitham and Richards (LWR) model and covers the full range of the fundamental diagram and transforms CTM in its entirety to a set of mixed-integer constraints.

Alecsandru [126] presents a series of enhancements to the original form of CTM such as topological enhancements and modifications to the flow advancing equation to allow variable cell lengths and nondiscrete movements of vehicles between cells and lane-changing behavior.

Zhong and Sumalee [127] develop a stochastic cell transmission model for the evolution of traffic flows on freeways taking into account various uncertainties. The model is formulated as a discrete time bilinear stochastic system.

Sumalee et al. [128] propose the stochastic cell transmission model, to model traffic flow density on freeway segments with stochastic demand and supply considering five operational modes corresponding to different congestion levels of the freeway segment, which are formulated as discrete time bilinear stochastic systems. The model captures the mean and standard deviation of density of the traffic flow and the propagation of it over time and space.

Carey et al. [129] consider a road link that consists of two adjacent homogeneous lanes and uses a modified cell transmission model to allow moving between lanes. The authors investigate how lane changing and congestion are affected by varying some behavioural parameters.

Multiclass Models. Szeto et al. [130] propose a cell-based multiclass dynamic traffic assignment problem that is formulated as a fixed point problem and considers the random evolution of traffic states, where travelers select routes based on perceived effective travel time, that is, the sum of mean perceived travel time and safety margin. The problem includes a MonteCarlo-based stochastic cell transmission model to capture the effect of physical queues and the random evolution of traffic states.

Tuerprasert and Aswakul [131] present a generalization of the cell transmission model to a more generalized multiclass cell transmission model taking into account the several vehicle classes to introduce head-of-cell and end-of-cell vehicles into the model. Cascading, merging, and diverging scenarios of cells allow the model to be applied to general road networks.

Variational Models. These models are represented mathematically by variational equations. Marcotte [132] deals with the variational inequality problem formulated as a nonconvex, nondifferentiable optimization problem, shows that any stationary point is optimal, and proposes an algorithm that decreases the nondifferentiable objective monotonically.

Zhu and Marcotte [133] deal with the existence of solutions to a dynamic network equilibrium problem modeled as an infinite dimensional variational inequality and introduce a novel concept that strengthens the familiar concept of FIFO.

Wang and Jiang [134] discuss the problem of traffic equilibrium in which the cost of route is not the simple sum of the cost of links and establish a variational inequality model to characterize the solutions of traffic equilibrium problems.

Lo and Szeto [135] present a cell-based dynamic traffic assignment formulation that follows the ideal dynamic user optimal (DUO) principle through a variational inequality approach, which encapsulates a network version of the cell transmission model (CTM) and satisfies the FIFO conditions through the CTM. The results showed that the formulation was capable of capturing shockwaves and queue formation and dissipation.

Bliemer and Bovy [136] consider multiple user classes in a macroscopic dynamic traffic assignment model. Considering different asymmetries, such as interuser-class interaction and interspatial and intertemporal asymmetries, the model is specified as a (quasi) variational inequality problem.

Network Loading Models. Network loading models allow simulating the propagation of traffic profiles through link and networks. To this end, several models have appeared in the existing literature.

Astarita [32] proposes a dynamic network loading model, which contrary to exit functions is based on travel times. Two requirements for a satisfactory model are identified: allowance for overtaking on a link and flow propagation consistent with speed. The FIFO rule, not satisfied in this case, and its implications are discussed.

Wu et al. [28] formulate the continuous dynamic network loading problem as a system of functional equations.

$\mathrm{Xu}$ et al. [137] present a formulation of the continuous dynamic network loading problem where travel delays may be nonlinear functions of arc traffic volumes and propose a finite-step algorithm to obtain solutions.

Astarita et al. [138] investigate three methods for dynamic network loading: link-based travel time functions, the cell 
transmission model, and a link-based model derived from a simplified car-following relationship.

Rubio-Ardanaz et al. [139] present two improved eventbased simulations numerical algorithms for the continuous dynamic network loading problem.

Dynamic conservation equations (16) and flow definition constraints (20), by means of $\theta_{a}^{r}(t)$ function, permit relating the flows at different locations and times to establish a flow balance under a FIFO assumption. These relations allow modelling the flow evolution through the network. For example, Castillo et al. [45] present a FIFO consistent model for the continuous dynamic network loading problem. The nonlinear link travel time functions are calculated at a finite set of times used to interpolate a monotone cubic spline for all times. The model consists of five units: route origin flow wave definition, route wave propagation, congestion analysis, flow propagation, and inference engine. Next, the individual route profiles are propagated throughout routes using a conservation equation that stretches or enlarges the wave lengths and heights, depending on the degree of congestion at different links. Then, the individual route profiles are combined together to generate the link and node profiles. An iterative method is used until convergence.

Genetic Algorithms. Since the resulting optimization problems are in some cases too complex, some authors suggest the use of genetic algorithms, such as Lo [39] and Lo and Szeto [140], who use these algorithms to solve dynamic traffic assignment problem.

Neural Network Models. Celikoglu [141] proposes a neural network approximator that supports the dynamic network loading model to reproduce link flow dynamics on a sample network.

Other Models. G. C. K. Wong and S. C. Wong [142] present an extension of the Lighthill, Whitham, and Richards model with heterogeneous drivers where faster vehicles can overtake slower ones under the uncongested condition and the congested condition and slower vehicles could slow down the faster ones.

Blumberg and Bar-Gera [143] aim to achieve consistent integration between the behavior of traffic at different roadway elements and the propagation of flows along routes focusing in particular on the order of arrival to nodes. The authors propose the concept of anticipated arrival order (AAO) using a traffic flow behavior that follows the kinematic wave model with a triangular flow-density relationship to describe the number of vehicles expected to choose a certain downstream link at a node as a function of the total number of vehicles at this node. An iterative model permits solving the problem.

Long et al. [144] develop new formulations for dynamic traffic assignment of the step function (SF) and linear interpolation (LI) link travel time models in which the profiles of cumulative flows are piecewise linearized and both models are used to approximate cumulative flows over time. One of the formulations ensures causality, strong FIFO, and travel time continuity.
Kalman Filter Models. Kalman filter models and extensions were proposed by Okutani and Stephanedes [145] who propose two models employing Kalman filtering theory for predicting short-term traffic volume; Barcelò et al. [114] extend the previous research on Kalman filtering approaches for freeway OD estimation to more complex topologies of urban networks where alternative route choices between origins and destinations are available.

\section{Conclusions}

In this paper a state-of-the-art review of observability together with estimation and prediction methods has been performed. The three problems have been analyzed considering them as optimization problems or their associated systems of equations. The roles of data, constraints, and objective functions or metrics have been discussed to embed the existing literature under an optimization paradigm.

The main conclusions of this paper are the following:

(1) The basic equations or constraints arise from the topology of the network, the different flow definitions, and nonnegativity or capacity constraints, which can be stated in a natural way because they must always hold.

(2) Data arise as the second fundamental information to solve the three stated problems. They can be direct observations obtained from passive or active counters or other means or prior information on previous traffic flows.

(3) Since the two previous data items are not sufficient and lead to infinitely many solutions, a metric must be used to select among all feasible solutions one that is considered as the best under the metric criterion. Selecting an adequate metric is crucial to have a good solution to the traffic problem.

(4) When the objective functions or metrics are not sufficient to provide unique solutions, a hierarchical objective function considering several criteria (metrics) can be used (e.g., using weights).

(5) While the observability and the flow estimation problems have been widely analyzed for the static case in the existing literature, these problems for the dynamic case still require a lot of attention. In particular, observability methods and estimation of $\tau_{a}(t)$ function that provides the link travel time functions together with traffic volumes at some locations are interesting topics for future research.

(6) Special care must be taken with redundancy because it can lead to the nonexistence of feasible solutions. In this case, least squares equations or similar equations should replace the direct observation equations to allow for possible errors.

(7) Use of dimensionless equations is much better than other alternatives. This has been illustrated with expression (8), devoted to generalized least squares, where, instead of absolute flow values, relative flow values were used. 
(8) In the static observability problem, algebraic tools, such as null spaces, are the basic tools to be used and the path information is crucial to obtain an important reduction in the number of links required for partial of complete link flow observability. Node based approaches provide interesting and easily obtainable links but they need not be sharp.

(9) The consideration of some model parameters, such as link choice proportions, as known values is not specially recommended, because they are large numbers and due to the difficulties in guessing realistic values.

(10) The use of cumulative flows in dynamic cases arises as a promising alternative that avoids the use of partial derivatives and permits replacing differential equations by functional equations. This alternative deserves further research.

(11) As indicated in this paper, passive counters provide a very limited and an insufficient amount of information. Thus, active counters must be used if relevant information about path information and good predictions are required.

(12) The optimal location of counters leads to important savings in cost and allows optimizing the resulting information. Several works dealing with this problem have been discussed.

(13) As indicated in Section 2.2.1, some constraints suggested for the dynamic case lead to contradiction and must be avoided.

(14) The FIFO rule plays an important role in building an important group of dynamic models but can be replaced by considering other types of models, such as multiclass models with overtaking possibilities.

(15) Finally, as indicated in this paper, two different classes of models, point and physical queue models, must be differentiated depending on the aim of the analysis.

\section{Notations}

$\mathscr{A}: \quad$ Set of counted links

$\mathscr{C}$ : $\quad$ Subset of counted links

$c p_{a}$ : $\quad$ Capacity of link $a$ per unit time

$c_{a}\left(v_{a}\right)$ : Link $a$ travel time function

$C_{a}\left(v_{a}\right)$ : Integral of the link $a$ travel time function

$d_{a}(t)$ : Travel time of a vehicle that enters link $a$ at time $t$

$f_{r}: \quad$ Flow on route $r$

$F_{r}(t)$ : Cumulated flow that has entered route $r$ at time $t$

$g_{a}(x)$ : Exit function giving the rate at which traffic exits link $a$ as a function of link traffic volume $x$

$h$ : $\quad$ Number of finite (bounded) holes

$\mathcal{I}_{n}: \quad$ Set of links starting at node $n$

$I_{n}(t)$ : Cumulated flow that has entered node $n$ at time $t$

$k_{\text {jam }}: \quad$ Jam density

$k$ : $\quad$ Flow density $k_{0}$ : Number of added links to convert the maximal planar network into a nonplanar hole-generated network

$n_{c}: \quad$ Number of cameras or sensors

$n_{c}^{\max }:$ Available number of cameras

$n_{\max }:$ Maximum number of sensors per route

$\mathscr{N}: \quad$ Set of nodes

$\mathscr{L}: \quad$ Set of links

O: $\quad$ Subset of observed flows

$\mathcal{O}_{n}: \quad$ Set of links ending at node $n$

$O_{n}(t)$ : Cumulated flow that has left node $n$ at time $t$

$p_{r w}(t)$ : Proportion of cumulated users choosing route $r$ in OD pair $w$ at time $t$

Q: $\quad$ Inflow capacity

$\mathscr{R}: \quad$ Set of routes

$\mathscr{R}_{\text {obs }}$ : Subset of routes that we want to observe

$r: \quad$ Route

$\mathscr{R}(w)$ : Set of routes in OD pair $w$

$\psi_{r}(t)$ : Travel time of route $r$ at time $t$

$\tau_{w}$ : $\quad$ Flow that has selected a route in OD pair $w$

$\mathscr{T}: \quad$ Set of selected times

$\mathscr{T}^{*}: \quad$ Set of times at which data are collected

$T_{w}(t)$ : Cumulated flow that has entered a route in OD pair $w$ at time $t$

$u_{a}(t):$ Rate of flow entering link $a$ at time $t$

$\mathscr{U}$ : $\quad$ Subset of unobserved flows

$U_{a}(t)$ : Cumulated flow at the entrance of link $a$ at time $t$

$v_{a}: \quad$ Flow on link $a$

$v_{a}^{\max }: \quad$ Practical flow capacity of link $a$

$\widehat{v}_{a}: \quad$ Counted (observed) flow on link $a$

$V: \quad$ Free-flow speed

$V_{a}^{i}(t)$ : Cumulated flow that has entered link $a$ at time $t$

$\widehat{V}_{a}^{i}(t)$ : Counted cumulated flow that has entered link $a$ at time $t$

$V_{a}^{o}(t)$ : Cumulated flow that has left link $a$ at time $t$

$w_{a}(t)$ : Rate of flow exiting link $a$ at time $t$

$\widehat{w}_{s}: \quad$ Flow associated with the plate scanned link combination $s$

$W: \quad$ Speed of the backward shock wave or backward disturbances propagation speed

$\mathscr{W}: \quad$ Set of OD pairs

$W_{a}(t)$ : Cumulated flow at the exit of link $a$ at time $t$

$\widehat{W}_{s}(t)$ : Total plate scanned observations in subset $s$ at time $t$

$x_{a}(t)$ : Traffic volume on link $a$ at time $t$

$y_{r}$ : Binary variable that equals one if route $r$ is observed and zero otherwise

$z_{a}: \quad$ Binary variable that equals one if link $a$ contains a sensor and zero otherwise

$\alpha: \quad$ Positive coefficient

$\delta_{r}^{a}: \quad$ Element of the link-route incidence matrix, which equals one if link $a$ is on route $r$ and equals zero otherwise

$\varepsilon: \quad$ Small dimensionless number

$\eta_{r}^{s}$ : $\quad$ Element of route-scanned combination incidence matrix for route $r$, which equals one if route $r$ contains the subset $s \subseteq \mathcal{S}$ of scanned links and no more scanned links and zero otherwise 
$\theta_{a}^{r}(t)$ : Entry time to link $a$ on route $r$ of a vehicle that enters route $r$ at time $t$

$\rho_{1}, \rho_{2}$ : Nonnegative numbers that measure the relative weight we give to the prior information of route and OD flows with respect to link flows

$\theta_{w}$ : Parameter that scales the perceived travel time in OD pair $w$

$\kappa: \quad$ Positive coefficient

$\kappa_{s}^{a}$ : $\quad$ Being one if link $a$ appears in the link combination $s$ and zero otherwise

$\boldsymbol{\omega}^{f}$ : Covariance matrix associated with route flows

$\boldsymbol{\omega}^{\ell}: \quad$ Covariance matrix associated with link flows

$\boldsymbol{\omega}^{\tau}$ : Covariance matrix associated with OD flows

$\rho_{a w}$ : Proportions of OD flow $\tau_{w}$ using link $a$

$\xi_{r w}$ : The element of the route-OD incidence matrix, which equals 1 if route $r$ belongs to OD pair $w$ and zero otherwise

$\tau_{a}: \quad$ Travel time on link $a$

$\tau_{a}(t)$ : Link $a$ travel time of a vehicle that enters the link at time $t$

$\tau_{0 a}: \quad$ Free-flow travel time on link $a$.

\section{Conflict of Interests}

The authors declare that there is no conflict of interests regarding the publication of this paper.

\section{References}

[1] V. P. Gil Jiménez and M. J. Fernández-Getino García, "Simple design of wireless sensor networks for traffic jams avoidance," Journal of Sensors, vol. 2015, Article ID 380794, 7 pages, 2015.

[2] C. Liu, H. Zheng, D. Yu, and X. Xu, "A novel method of adaptive traffic image enhancement for complex environments," Journal of Sensors, vol. 2015, Article ID 516326, 9 pages, 2015.

[3] W. Kong, L. Zhou, Y. Wang, J. Zhang, J. Liu, and S. Gao, "A system of driving fatigue detection based on machine vision and its application on smart device," Journal of Sensors, vol. 2015, Article ID 548602, 11 pages, 2015.

[4] M. Gentili and P. B. Mirchandani, "Locating active sensors on traffic networks," Annals of Operations Research, vol. 136, no. 1, pp. 229-257, 2005.

[5] D. Branston, "Link capacity functions: a review," Transportation Research Part B, vol. 10, no. 4, pp. 223-236, 1976.

[6] T. Abrahamsson, "Estimation of origin-destination matrices using traffic counts literature survey," Interim Report IR98-021, International institute for Applied System Analysis, Computer Science Press, Laxenburg City, Laxenburg, 1998.

[7] R. Montoya-Zamora, J. A. Romero, and R. Guzmán-Cruz, "Comparison between heuristics and methodologies to solve the NSLP," International Journal of Engineering Science and Technology, vol. 3, no. 7, pp. 5929-5939, 2011.

[8] R. M. Li, S. M. Tang, and F. Y. Wang, "A review of dynamic user-optimal variational inequality traffic assignment," Journal of Transportation Systems Engineering and Information Technology, vol. 6, no. 2, pp. 83-90, 2006.

[9] S. Peeta and A. K. Ziliaskopoulos, "Foundations of dynamic traffic assignment: the past, the present and the future," Networks and Spatial Economics, vol. 1, no. 3-4, pp. 233-265, 2001.
[10] D. Boyce, D.-H. Lee, and B. Ran, "Analytical models of the dynamic traffic assignment problem," Networks and Spatial Economics, vol. 1, no. 3-4, pp. 377-390, 2001.

[11] W. Y. Szeto and H. K. Lo, "Dynamic traffic assignment: review and future," Journal of Transportation Systems Engineering and Information Technology, vol. 5, pp. 85-100, 2005.

[12] E. Castillo, M. Nogal, A. Rivas, and S. Sánchez-Cambronero, "Observability of traffic networks: optimal location of counting and scanning devices," Transportmetrica B: Transport Dynamics, vol. 1, no. 1, pp. 68-102, 2013.

[13] H. Yang and J. Zhou, "Optimal traffic counting locations for origin-destination matrix estimation," Transportation Research-Part B: Methodological, vol. 33, no. 2, pp. 109-126, 1998.

[14] M. Gentili and P. B. Mirchandani, "Locating sensors on traffic networks: models, challenges and research opportunities," Transportation Research Part C: Emerging Technologies, vol. 24, pp. 227-255, 2012.

[15] E. Cascetta, Transportation Systems Engineering: Theory and Methods, Kluwer Academic Publishers, Boston, Mass, USA, 2001.

[16] A. Papola and V. Marzano, "How can we trust in the OD matrix correction procedure using traffic counts?" in Proceedings of the European Transport Conference (ETC '06), Strasbourg, France, 2006.

[17] E. Cascetta, "Estimation of trip matrices from traffic counts and survey data: a generalized least squares estimator," Transportation Research Part B, vol. 18, no. 4-5, pp. 289-299, 1984.

[18] E. Cascetta and S. Nguyen, "A unified framework for estimating or updating origin/destination matrices from traffic counts," Transportation Research Part B, vol. 22, no. 6, pp. 437-455, 1988.

[19] J. Doblas and F. G. Benítez, "An approach to estimating and updating origin-destination matrices based upon traffic counts preserving the prior structure of a survey matrix," Transportation Research Part B: Methodological, vol. 39, no. 7, pp. 565-591, 2005.

[20] S. C. Wong, C. O. Tong, K. I. Wong et al., "Estimation of multiclass origin-destination matrices from traffic counts," Journal of Urban Planning and Development, vol. 131, no. 1, pp. 19-29, 2005.

[21] E. Castillo, J. M. Menéndez, and S. Sánchez-Cambronero, “Traffic estimation and optimal counting location without path enumeration using Bayesian networks," Computer-Aided Civil and Infrastructure Engineering, vol. 23, no. 3, pp. 189-207, 2008.

[22] R. Mínguez, S. Sánchez-Cambronero, E. Castillo, and P. Jiménez, "Optimal traffic plate scanning location for OD trip matrix and route estimation in road networks," Transportation Research Part B: Methodological, vol. 44, no. 2, pp. 282-298, 2010.

[23] X. P. Li and Y. F. Ouyang, "Reliable sensor deployment for network traffic surveillance," Transportation Research-Part B: Methodological, vol. 45, no. 1, pp. 218-231, 2011.

[24] T. L. Friesz, D. Bernstein, N. J. Mehta, R. L. Tobin, and S. Ganjalizadeh, "Day-to-day dynamic network disequilibria and idealized traveler information systems," Operations Research, vol. 42, no. 6, pp. 1120-1136, 1994.

[25] G. E. Cantarella and E. Cascetta, "Dynamic processes and equilibrium in transportation networks: towards a unifying theory," Transportation Science, vol. 29, no. 4, pp. 305-329, 1995.

[26] H. K. Lo and W. Y. Szeto, "Modeling advanced traveler information services: static versus dynamic paradigms," Transportation 
Research Part B: Methodological, vol. 38, no. 6, pp. 495-515, 2004.

[27] E. Cascetta, D. Inaudi, and G. Marquis, "Dynamic estimators of origin-destination matrices using traffic counts," Transportation Science, vol. 27, no. 4, pp. 363-373, 1993.

[28] J. H. Wu, Y. Chen, and M. Florian, “The continuous dynamic network loading problem: a mathematical formulation and solution method," Transportation Research-Part B: Methodological, vol. 32, no. 3, pp. 173-187, 1998.

[29] E. Castillo, M. Nogal, J. M. Menéndez, S. Sánchez-Cambronero, and P. Jiménez, "Stochastic demand dynamic traffic models using generalized beta-gaussian bayesian networks," IEEE Transactions on Intelligent Transportation Systems, vol. 13, no. 2, pp. 565-581, 2012.

[30] M. Bierlaire, "The total demand scale: a new measure of quality for static and dynamic origin-destination trip tables," Transportation Research Part B, vol. 36, no. 9, pp. 837-850, 2002.

[31] D. K. Merchant and G. L. Nemhauser, "Optimality conditions for a dynamic traffic assignment model," Transportation Science, vol. 12, no. 3, pp. 200-207, 1978.

[32] V. Astarita, "A continuous time link model for dynamic network loading based on travel time function," in Transportation and Traffic Theory: Proceedings of the 13th International Symposium on Transportation and Traffic Theory (ISTTT '96), J. B. Lesort, Ed., pp. 79-101, ISTTT, Lyon, France, 1996.

[33] T. L. Friesz, D. Bernstein, T. E. Smith, R. L. Tobin, and B. W. Wie, "A variational inequality formulation of the dynamic network user equilibrium problem," Operations Research, vol. 41, no. 1, pp. 179-191, 1993.

[34] J. E. Fernández and J. De Cea, "Flow propagation description in dynamic network assignment models," in Proceedings of the 2nd Triennal Symposium on Transportation Analysis (TRISTAN '94), pp. 517-531, Capri, Italy, June 1994.

[35] J. H. Wu, Y. Chen, and M. Florian, "The continuous dynamic network loading problem: a mathematical formulation and solution method," in Proceedings of the 3rd Euro Working Group Meeting on Urban Traffic and Transportation, Barcelona, Spain, 1995.

[36] C. F. Daganzo, "The cell transmission model: a dynamic representation of highway traffic consistent with the hydrodynamic theory," Transportation Research Part B, vol. 28, no. 4, pp. 269287, 1994.

[37] C. F. Daganzo, “The cell transmission model, part II: network traffic," Transportation Research Part B: Methodological, vol. 29, no. 2, pp. 79-93, 1995.

[38] W. Y. Szeto and H. K. Lo, "A cell-based simultaneous route and departure time choice model with elastic demand," Transportation Research Part B: Methodological, vol. 38, no. 7, pp. 593-612, 2004.

[39] H. K. Lo, "A dynamic traffic assignment formulation that encapsulates the cell transmission model," in Transportation and Traffic Theory, A. Ceder, Ed., pp. 327-350, 1999.

[40] J. M. Rubio-Ardanaz, J. H. Wu, and M. A. Florian, "A numerical analytical model for the continuous dynamic network equilibrium problem with limited capacity and spill back," in Proceedings of the IEEE Intelligent Transportation Systems Conference, pp. 263-267, Oakland, Calif, USA, August 2001.

[41] M. Kuwahara and T. Akamatsu, "Dynamic user optimal assignment with physical queues for a many-to-many OD pattern," Transportation Research Part B: Methodological, vol. 35, no. 5, pp. 461-479, 2001.
[42] H. K. Lo and W. Y. Szeto, "Road pricing modeling for hypercongestion," Transportation Research Part A: Policy and Practice, vol. 39, no. 7-9, pp. 705-722, 2005.

[43] M. Nogal, Mathematical methods for traffic prediction [Ph.D. thesis], University of Cantabria, Santander, Spain, 2011.

[44] A. Calviño, Some statistical and mathematical tools for traffic modeling [Ph.D. thesis], University of Cantabria, Santander, Spain, 2013.

[45] E. Castillo, J. M. Menéndez, M. Nogal, P. Jiménez, and S. Sánchez-Cambronero, "A FIFO rule consistent model for the continuous dynamic network loading problem," IEEE Transactions on Intelligent Transportation Systems, vol. 13, no. 1, pp. 264283, 2012.

[46] G. Strang, Introduction to Applied Mathematics, WellesleyCambridge Press, Wellesley, Mass, USA, 1986.

[47] A. Ehlert, M. G. H. Bell, and S. Grosso, "The optimisation of traffic count locations in road networks," Transportation Research Part B: Methodological, vol. 40, no. 6, pp. 460-479, 2006.

[48] H. D. Sherali, N. Arora, and A. G. Hobeika, "Parameter optimization methods for estimating dynamic origin-destination trip-tables," Transportation Research-Part B: Methodological, vol. 31, no. 2, pp. 141-157, 1997.

[49] S. M. Eisenman, X. Fei, X. Zhou, and H. Mahmassani, "Number and location of sensors for realtime network traffic estimation and prediction: a sensitivity analysis," TRB Paper 06-2408, Transportation Research Board CD-ROM Paper Preprints, 2006.

[50] E. Castillo, J. M. Menéndez, and P. Jiménez, “Trip matrix and path flow reconstruction and estimation based on plate scanning and link observations," Transportation Research Part B: Methodological, vol. 42, no. 5, pp. 455-481, 2008.

[51] H. Yang, Y. Iida, and T. Sasaki, "An analysis of the reliability of an origin-destination trip matrix estimated from traffic counts," Transportation Research-Part B, vol. 25, no. 5, pp. 351-363, 1991.

[52] F. Viti, C. Tampere, W. Verbeke, and B. Immers, "Sensor locations for reliable travel time prediction and dynamic management of traffic networks," Transportation Research Record, vol. 2049, pp. 103-110, 2008.

[53] A. Chen, P. Chootinan, and W. W. Recker, "Examining the quality of synthetic origin-destination trip table estimated by path flow estimator," Journal of Transportation Engineering, vol. 131, no. 7, pp. 506-513, 2005.

[54] L. Gan, H. Yang, and S. C. Wong, "Traffic counting location and error bound in origin-destination matrix estimation problems," Journal of Transportation Engineering, vol. 131, no. 7, pp. 524534, 2005.

[55] M. Gentili, New models and algorithms for the location of sensors on traffic networks [Ph.D. thesis], Dipartimento di Statistica, Probabilità e Statistiche Applicate, Università di Roma "La Sapienza", Roma, Italy, 2002.

[56] E. Castillo, P. Jiménez, J. M. Menéndez, and A. J. Conejo, "The observability problem in traffic models: algebraic and topological methods," IEEE Transactions on Intelligent Transportation Systems, vol. 9, no. 2, pp. 275-287, 2008.

[57] M. W. Ng, "Synergistic sensor location for link flow inference without path enumeration: a node-based approach," Transportation Research-Part B: Methodological, vol. 46, no. 6, pp. 781-788, 2012. 
[58] M. W. Ng, "Partial link flow observability in the presence of initial sensors: solution without path enumeration," Transportation Research-Part E: Logistics and Transportation Review, vol. 51, pp. 62-66, 2013.

[59] S.-X. He, "A graphical approach to identify sensor locations for link flow inference," Transportation Research Part B: Methodological, vol. 51, pp. 65-76, 2013.

[60] E. Castillo, A. Rivas, P. Jiménez, and J. M. Menéndez, "Observability in traffic networks. Plate scanning added by counting information," Transportation, vol. 39, no. 6, pp. 1301-1333, 2012.

[61] H. Spiess, "A maximum likelihood model for estimating origindestination matrices," Transportation Research-Part B: Methodological, vol. 21, no. 5, pp. 395-412, 1987.

[62] N. L. Nihan and G. A. Davis, "Application of prediction-error minimization and maximum likelihood to estimate intersection O-D matrices from traffic counts," Transportation Science, vol. 23, no. 2, pp. 77-90, 1989.

[63] Y. Vardi, "Network tomography: estimating source-destination traffic intensities from link data," Journal of the American Statistical Association, vol. 91, no. 433, pp. 365-377, 1996.

[64] K. Parry and M. L. Hazelton, "Estimation of origin-destination matrices from link counts and sporadic routing data," Transportation Research-Part B: Methodological, vol. 46, no. 1, pp. 175-188, 2012.

[65] H. J. Van Zuylen and L. G. Willumsen, "The most likely trip matrix estimated from traffic counts," Transportation Research Part B: Methodological, vol. 14, no. 3, pp. 281-293, 1980.

[66] X. Zhou and G. F. List, "An information-theoretic sensor location model for traffic origin-destination demand estimation applications," Transportation Science, vol. 44, no. 2, pp. 254-273, 2010.

[67] F. Simonelli, V. Marzano, A. Papola, and I. Vitiello, "A network sensor location procedure accounting for o-d matrix estimate variability," Transportation Research-Part B: Methodological, vol. 46, no. 10, pp. 1624-1638, 2012.

[68] P. K. N. Yim and W. H. K. Lam, "Evaluation of count location selection methods for estimation of O-D matrices," Journal of Transportation Engineering, vol. 124, no. 4, pp. 376-382, 1998.

[69] X. Fei and H. S. Mahmassani, "Structural analysis of nearoptimal sensor locations for a stochastic large-scale network," Transportation Research Part C: Emerging Technologies, vol. 19, no. 3, pp. 440-453, 2011.

[70] F. Viti and F. Corman, "A novel approach to the sensor location problem for measuring the observed network flow variability," in Proceedings of the 5th International Symposium of Traffic Network Reliability, Hong Kong, December 2012.

[71] F. Viti, M. Rinaldi, F. Corman, and C. M. J. Tampèreb, "Assessing partial observability in network sensor location problems," Transportation Research-Part B: Methodological, vol. 70, pp. 65-89, 2014.

[72] E. Castillo, A. Calviño, J. M. Menéndez, M. P. Jiménez, and A. Rivas, "Deriving the upper bound of the number of sensors required to know all link flows in a traffic network," IEEE Transactions on Intelligent Transportation Systems, vol. 14, no. 2, pp. 761-771, 2013.

[73] E. Castillo, A. Calviño, H. K. Lo, J. M. Menéndez, and Z. Grande, "Non-planar hole-generated networks and link flow observability based on link counters," Transportation Research Part B: Methodological, vol. 68, pp. 239-261, 2014.

[74] S.-R. Hu, S. Peeta, and C.-H. Chu, "Identification of vehicle sensor locations for link-based network traffic applications,"
Transportation Research Part B: Methodological, vol. 43, no. 89, pp. 873-894, 2009.

[75] E. Castillo, A. Cobo, F. Jubete, and R. Pruneda, Orthogonal Sets and Polar Methods in Linear Algebra: Applications to Matrix Calculations, Systems of Equations, Inequalities, and Linear Programming, John Wiley \& Sons, New York, NY, USA, 1999.

[76] E. Castillo, A. Cobo, F. Jubete, R. Pruneda, and C. Castillo, "An orthogonally based pivoting transformation of matrices and some applications," SIAM Journal on Matrix Analysis and Applications, vol. 22, no. 3, pp. 666-681, 2000.

[77] E. Castillo, I. Gallego, J. M. Menéndez, and P. Jiménez, "Link flow estimation in traffic networks on the basis of link flow observations," Journal of Intelligent Transportation Systems: Technology, Planning, and Operations, vol. 15, no. 4, pp. 205-222, 2011.

[78] H. Mori and S. Tsuzuki, "A fast method for topological observability analysis using a minimum spanning tree technique," IEEE Transactions on Power Systems, vol. 6, no. 2, pp. 491-500, 1991.

[79] E. Castillo, F. Jubete, R. E. Pruneda, and C. Solares, “Obtaining simultaneous solutions of linear subsystems of inequalities and duals," Linear Algebra and Its Applications, vol. 346, no. 1-3, pp. 131-154, 2002.

[80] E. Castillo, A. Cobo, F. Jubete, R. E. Pruneda, and C. Castillo, "An orthogonally based pivoting transformation of matrices and some applications," SIAM Journal on Matrix Analysis and Applications, vol. 22, no. 3, pp. 666-681, 2000.

[81] M. J. Hodgson, "A flow-capturing location-allocation model," Geographical Analysis, vol. 22, no. 3, pp. 270-279, 1990.

[82] D. R. Morrison and S. E. Martonosi, "Characteristics of optimal solutions to the sensor location problem," Annals of Operations Research, 2014.

[83] E. Castillo, A. J. Conejo, J. M. Menéndez, and P. Jiménez, “The observability problem in traffic network models," ComputerAided Civil and Infrastructure Engineering, vol. 23, no. 3, pp. 208-222, 2008.

[84] E. Castillo, I. Gallego, S. Sánchez-Cambronero, and A. Rivas, "Matrix tools for general observability analysis in traffic networks," IEEE Transactions on Intelligent Transportation Systems, vol. 11, no. 4, pp. 799-813, 2010.

[85] M. Rinaldi, F. Corman, and F. Viti, "A null space metric for the analysis of partial network observability in sensor location problems," in Proceedings of the Annual Meeting of the Transportation Board, Washington, DC, USA, January 2013.

[86] E. Castillo, P. Jiménez, J. M. Menéndez, A. Rivas, and I. Gallego, "A ternary-arithmetic topological based algebraic method for networks traffic observability," Applied Mathematical Modelling, vol. 35, no. 11, pp. 5338-5354, 2011.

[87] W. H. K. Lam and H. P. Lo, "Accuracy of od estimates from traffic counting stations," Traffic Engineering and Control, vol. 7, no. 1, pp. 105-114, 1990.

[88] M. Bierlaire and P. L. Toint, "Meuse: an origin-destination matrix estimator that exploits structure," Transportation Research Part B, vol. 29, no. 1, pp. 47-60, 1995.

[89] Y. Asakura, E. Hato, and M. Kashiwadani, "Origin-destination matrices estimation model using automatic vehicle identification data and its application to the Han-Shin expressway network," Transportation, vol. 27, no. 4, pp. 419-438, 2000.

[90] V. Marzano, A. Papola, and F. Simonelli, "Limits and perspectives of effective od matrix correction using traffic counts," Transportation Research Part C: Emerging Technologies, vol. 17, no. 2, pp. 120-132, 2009. 
[91] M. G. Bell, "The estimation of an origin-destination matrix from traffic counts," Transportation Science, vol. 17, no. 2, pp. 198-217, 1983.

[92] D. P. Watling and M. J. Maher, "A statistical procedure for estimating a mean origin-destination matrix from a partial registration plate survey," Transportation Research-Part B, vol. 26, no. 3, pp. 171-193, 1992.

[93] D. P. Watling, "Maximum likelihood estimation of an origindestination matrix from a partial registration plate survey," Transportation Research-Part B, vol. 28, no. 4, pp. 289-314, 1994.

[94] H.-P. Lo and C.-P. Chan, "Simultaneous estimation of an origindestination matrix and link choice proportions using traffic counts," Transportation Research Part A: Policy and Practice, vol. 37, no. 9, pp. 771-788, 2003.

[95] R. Church and C. ReVelle, "The maximal covering location problem," Papers of the Regional Science Association, vol. 32, no. 1, pp. 101-118, 1974.

[96] X. Fei, H. S. Mahmassani, and S. M. Eisenman, "Sensor coverage and location for real-time traffic prediction in largescale networks," Transportation Research Record: Journal of the Transportation Research Board, vol. 2039, pp. 1-15, 2007.

[97] T. Larsson, J. T. Lundgren, and A. Peterson, "Allocation of link flow detectors for origin-destination matrix estimationa comparative study," Computer-Aided Civil and Infrastructure Engineering, vol. 25, no. 2, pp. 116-131, 2010.

[98] N. Wang, M. Gentili, and P. Mirchandani, "Model to locate sensors for estimation of static origin-destination volumes given prior flow information," Transportation Research Record: Journal of the Transportation Research Board, vol. 2283, pp. 6773, 2012.

[99] A. Chen, S. Pravinvongvuth, P. Chootinan, M. Lee, and W. Recker, "Strategies for selecting additional traffic counts for improving O-D trip table estimation," Transportmetrica, vol. 3 , no. 3, pp. 191-211, 2007.

[100] M. Brenninger-Göthe, K. O. Jörnsten, and J. T. Lundgren, "Estimation of origin-destination matrices from traffic counts using multiobjective programming formulations," Transportation Research Part B, vol. 23, no. 4, pp. 257-269, 1989.

[101] M. J. Maher, "Inferences on trip matrices from observations on link volumes: a Bayesian statistical approach," Transportation Research: Part B, vol. 17, no. 6, pp. 435-447, 1983.

[102] E. Castillo, P. Jiménez, J. M. Menéndez, and M. Nogal, “A Bayesian method for estimating traffic flows based on plate scanning," Transportation, vol. 40, no. 1, pp. 173-201, 2013.

[103] E. Castillo, J. M. Menéndez, S. Sánchez-Cambronero, A. Calviño, and J. M. Sarabia, "A hierarchical optimization problem: estimating traffic flow using gamma random variables in a Bayesian context," Computers \& Operations Research, vol. 41, no. 1, pp. 240-251, 2014.

[104] H. P. Lo, N. Zhang, and W. H. K. Lam, "Estimation of an origindestination matrix with random link choice proportions: a statistical approach," Transportation Research-Part B: Methodological, vol. 30, no. 4, pp. 309-324, 1996.

[105] E. Castillo, I. Gallego, J. M. Menéndez, and A. Rivas, “Optimal use of plate-scanning resources for route flow estimation in traffic networks," IEEE Transactions on Intelligent Transportation Systems, vol. 11, no. 2, pp. 380-391, 2010.

[106] S. Sánchez-Cambronero, E. Castillo, J. M. Menéndez, and P. Jiménez, "Dealing with error recovery in traffic flow prediction using Bayesian networks based on license plate scanning data,"
Journal of Transportation Engineering, vol. 137, no. 9, pp. 615629, 2011.

[107] H. Yang, C. Yang, and L. Gan, "Models and algorithms for the screen line-based traffic-counting location problems," Computers and Operations Research, vol. 33, no. 3, pp. 836-858, 2006.

[108] P. Chootinan, A. Chen, and H. Yang, "A bi-objective traffic counting location problem for origin-destination trip table estimation," Transportmetrica, vol. 1, no. 1, pp. 65-80, 2005.

[109] E. Cipriani, G. Fusco, S. Gori, and M. Petrelli, "Heuristic methods for the optimal location of road traffic monitoring," in Proceedings of the IEEE Intelligent Transportation Systems Conference (ITSC '06), pp. 1072-1077, Toronto, Canada, 2006.

[110] N. L. Nihan and G. A. Davis, "Recursive estimation of origindestination matrices from input/output counts," Transportation Research Part B: Methodological, vol. 21, no. 2, pp. 149-163, 1987.

[111] K. Ashok and M. E. Ben-Akiva, "Dynamic od matrix estimation and prediction for real-time traffic management systems," in International Symposium on the Theory of Traffic Flow and Transportation (12th: 1993: Berkeley, Calif.). Transportation and Traffic Theory, C. E. Daganzo, Ed., pp. 465-484, Elsevier, Amsterdam, The Netherlands, 1993.

[112] K. Ashok and M. E. Ben-Akiva, "Estimation and prediction of time-dependent origin-destination flows with a stochastic mapping to path flows and link flows," Transportation Science, vol. 36, no. 2, pp. 184-198, 2002.

[113] A. Peterson, The Origin-Destination Matrix Estimation Problem: Analysis and Computations, Institutionen för Teknik och Naturvetenskap, 2007.

[114] J. Barcelò, L. Montero, L. Marqués, and C. Carmona, "Ict based estimation of time dependent od matrices," in Proceedings of the 90th TRB Annual Meeting, Washington, DC, USA, January 2011.

[115] D. Boyce and B. Janson, "The effect on equilibrium trim assignment of different link congestion functions," Transportation Research Part B, vol. 15, pp. 223-232, 1981.

[116] M. G. H. Bell, "Alternatives to Dial's logit assignment algorithm," Transportation Research Part B, vol. 29, no. 4, pp. 287-295, 1995.

[117] E. Castillo, A. Calviño, S. Sánchez-Cambronero, and H. K. Lo, "A multiclass user equilibrium model considering overtaking across classes," IEEE Transactions on Intelligent Transportation Systems, vol. 14, no. 2, pp. 928-942, 2013.

[118] E. Castillo, M. Nogal, A. Calviño, A. Rivas, and H. K. Lo, “A model for continuous dynamic network loading problem with different overtaking class users," Journal of Intelligent Transportation Systems: Technology, Planning, and Operations, vol. 17, no. 4, pp. 328-350, 2013.

[119] E. Castillo, A. Calviño, S. Sánchez-Cambronero, M. Nogal, and A. Rivas, "A percentile system optimization approach with and without path enumeration," Computers \& Operations Research, vol. 40, no. 11, pp. 2711-2723, 2013.

[120] T. L. Friesz, J. Luque, R. L. Tobin, and B.-W. Wie, "Dynamic network traffic assignment considered as a continuous time optimal control problem," Operations Research, vol. 37, no. 6, pp. 893-901, 1989.

[121] R. Jayakrishnan, W. K. Tsai, and A. Chen, "A dynamic traffic assignment model with traffic-flow relationships," Transportation Research Part C: Emerging Technologies, vol. 3, no. 1, pp. 5172, 1995.

[122] W. Y. Szeto and H. K. Lo, "Dynamic traffic assignment: properties and extensions," Transportmetrica, vol. 2, no. 1, pp. 31-52, 2006. 
[123] M. Carey and E. Subrahmanian, "An approach to modelling time-varying flows on congested networks," Transportation Research Part B: Methodological, vol. 34, no. 3, pp. 157-183, 2000.

[124] C. F. Daganzo, “The cell-transmission model. Part I: a simple dynamic representation of highway traffic," Research Report UCBITS-PRR-93-7, Institute of Transportation Studies, University of California, Berkeley, Calif, USA, 1993.

[125] C. F. Daganzo, "A finite difference approximation of the kinematic wave model of traffic flow," Transportation Research Part B: Methodological, vol. 29, no. 4, pp. 261-276, 1995.

[126] C. D. Alecsandru, A stochastic mesoscopic cell-transmission model for operational analysis of large-scale transportation networks [Ph.D. thesis], Department of Civil and Environmental Engineering, Louisiana State University, 2006.

[127] R. Zhong and A. Sumalee, "Stochastic cell transmission model: traffic state estimation under uncertainties," in Traffic and Transportation Studies: Proceedings of the Sixth International Conference on Traffic and Transportation Studies, B. Mao, Z. Tian, H. Huang, and Z. E. Gao, Eds., pp. 462-478, ASCE, Reston, Va, USA, 2008.

[128] A. Sumalee, R. X. Zhong, T. L. Pan, and W. Y. Szeto, "Stochastic cell transmission model (SCTM): a stochastic dynamic traffic model for traffic state surveillance and assignment," Transportation Research-Part B: Methodological, vol. 45, no. 3, pp. 507533, 2011.

[129] M. Carey, C. Balijepalli, and D. Watling, "Extending the cell transmission model to multiple lanes and lane-changing," Networks and Spatial Economics, 2013.

[130] W. Y. Szeto, Y. Jiang, and A. Sumalee, "A cell-based model for multi-class doubly stochastic dynamic traffic assignment," Computer-Aided Civil and Infrastructure Engineering, vol. 26, no. 8, pp. 595-611, 2011.

[131] K. Tuerprasert and C. Aswakul, "Multiclass cell transmission model for heterogeneous mobility in general topology of road network," Journal of Intelligent Transportation Systems: Technology, Planning, and Operations, vol. 14, no. 2, pp. 68-82, 2010.

[132] P. Marcotte, "A new algorithm for solving variational inequalities with application to the traffic assignment problem," Mathematical Programming, vol. 33, no. 3, pp. 339-351, 1985.

[133] D. Zhu and P. Marcotte, "On the existence of solutions to the dynamic user equilibrium problem," Transportation Science, vol. 34, no. 4, pp. 402-414, 2000.

[134] J. Wang and Q. Jiang, "A variational inequality model for traffic assignment," in Proceedings of the International Conference on Internet Technology and Applications (iTAP '11), Wuhan, China, August 2011.

[135] H. K. Lo and W. Y. Szeto, "A cell-based variational inequality formulation of the dynamic user optimal assignment problem," Transportation Research Part B: Methodological, vol. 36, no. 5, pp. 421-443, 2002.

[136] M. C. J. Bliemer and P. H. L. Bovy, "Quasi-variational inequality formulation of the multiclass dynamic traffic assignment problem," Transportation Research Part B, vol. 37, no. 6, pp. 501-519, 2003.

[137] Y. W. Xu, J. H. Wu, M. Florian, P. Marcotte, and D. L. Zhu, "Advances in the continuous dynamic network loading problem," Transportation Science, vol. 33, no. 4, pp. 341-353, 1999.

[138] V. Astarita, K. Er-Rafia, M. Florian, M. Mahut, and S. Velan, "Comparison of three methods for dynamic network loading," Transportation Research Record, vol. 1771, pp. 179-190, 2001.
[139] J. M. Rubio-Ardanaz, J. H. Wu, and M. Florian, “Two improved numerical algorithms for the continuous dynamic network loading problem," Transportation Research-Part B: Methodological, vol. 37, no. 2, pp. 171-190, 2003.

[140] H. K. Lo and W. Y. Szeto, "A cell-based dynamic traffic assignment model: formulation and properties," Mathematical and Computer Modelling, vol. 35, no. 7-8, pp. 849-865, 2002.

[141] H. B. Celikoglu, "A dynamic network loading model for traffic dynamics modeling," IEEE Transactions on Intelligent Transportation Systems, vol. 8, no. 4, pp. 575-583, 2007.

[142] G. C. K. Wong and S. C. Wong, "A multi-class traffic flow model: an extension of LWR model with heterogeneous drivers," Transportation Research Part A: Policy and Practice, vol. 36, no. 9, pp. 827-841, 2002.

[143] M. Blumberg and H. Bar-Gera, "Consistent node arrival order in dynamic network loading models," Transportation Research Part B: Methodological, vol. 43, no. 3, pp. 285-300, 2009.

[144] J. Long, Z. Gao, and W. Y. Szeto, "Discretised link travel time models based on cumulative flows: formulations and properties," Transportation Research Part B: Methodological, vol. 45, no. 1, pp. 232-254, 2011.

[145] I. Okutani and Y. J. Stephanedes, "Dynamic prediction of traffic volume through Kalman filtering theory," Transportation Research-Part B: Methodological, vol. 18, no. 1, pp. 1-11, 1984. 

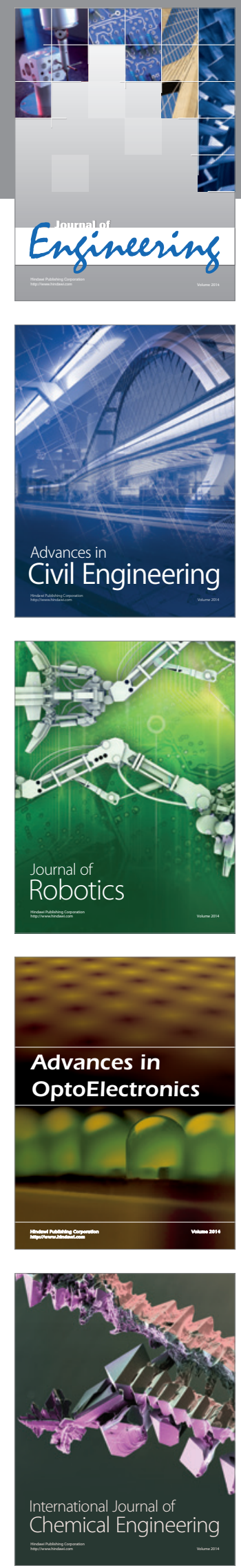

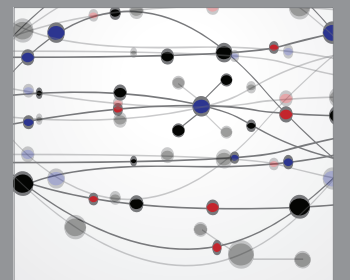

The Scientific World Journal
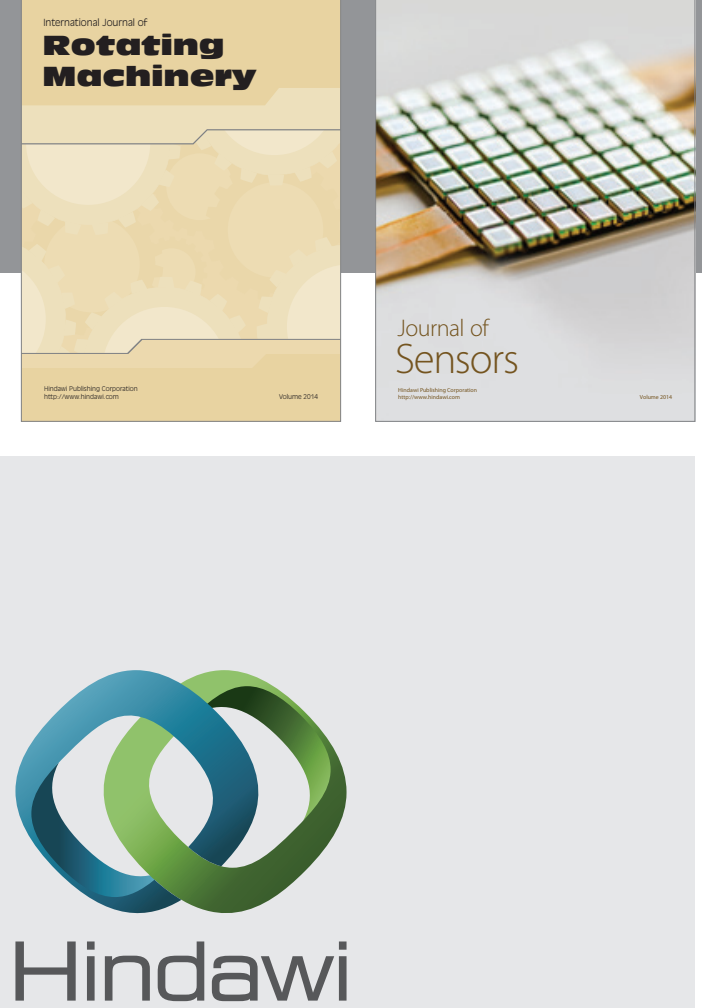

Submit your manuscripts at http://www.hindawi.com
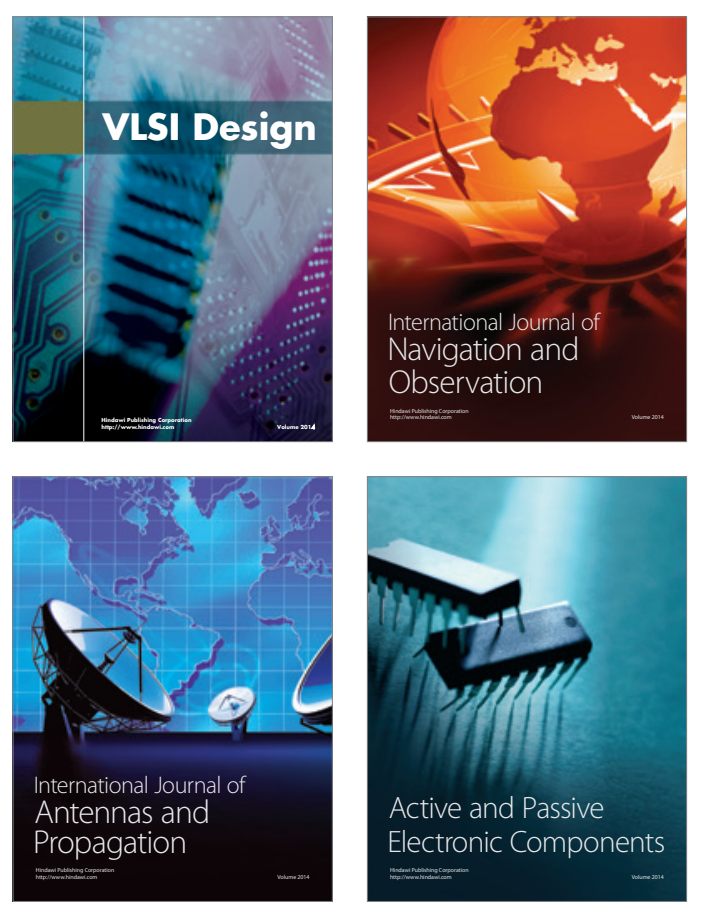
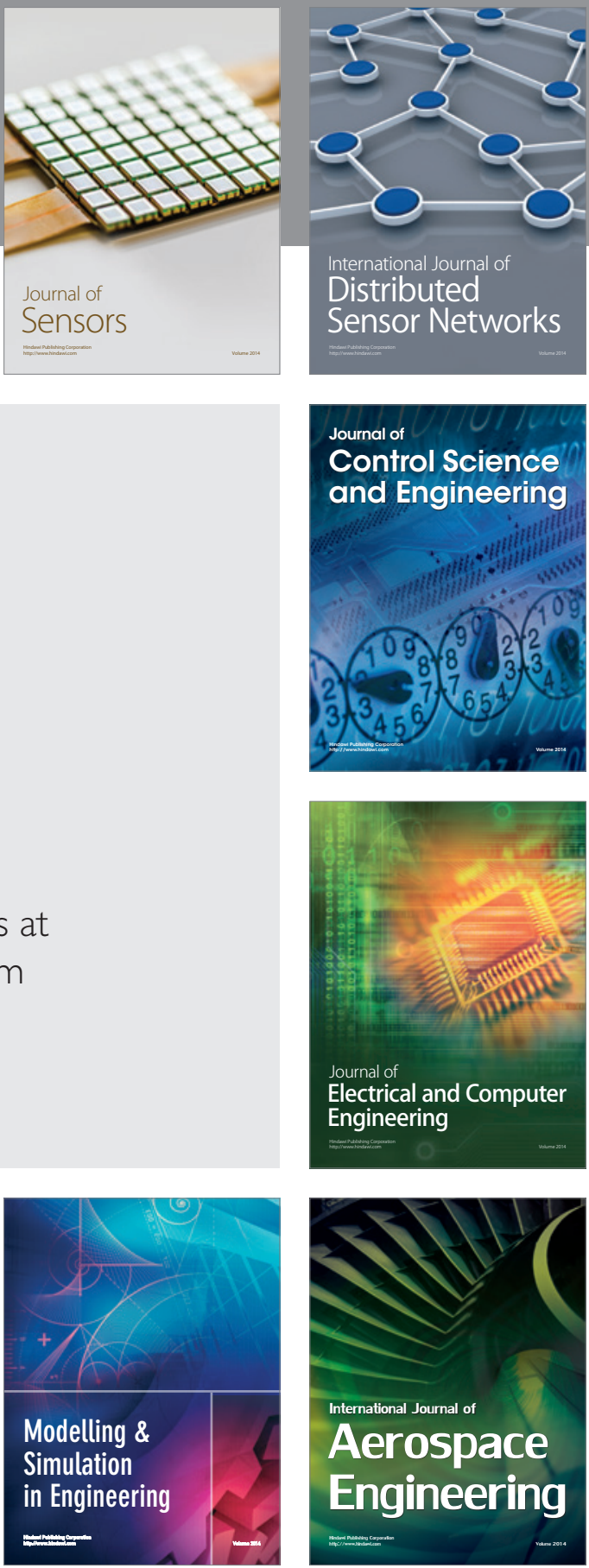

Journal of

Control Science

and Engineering
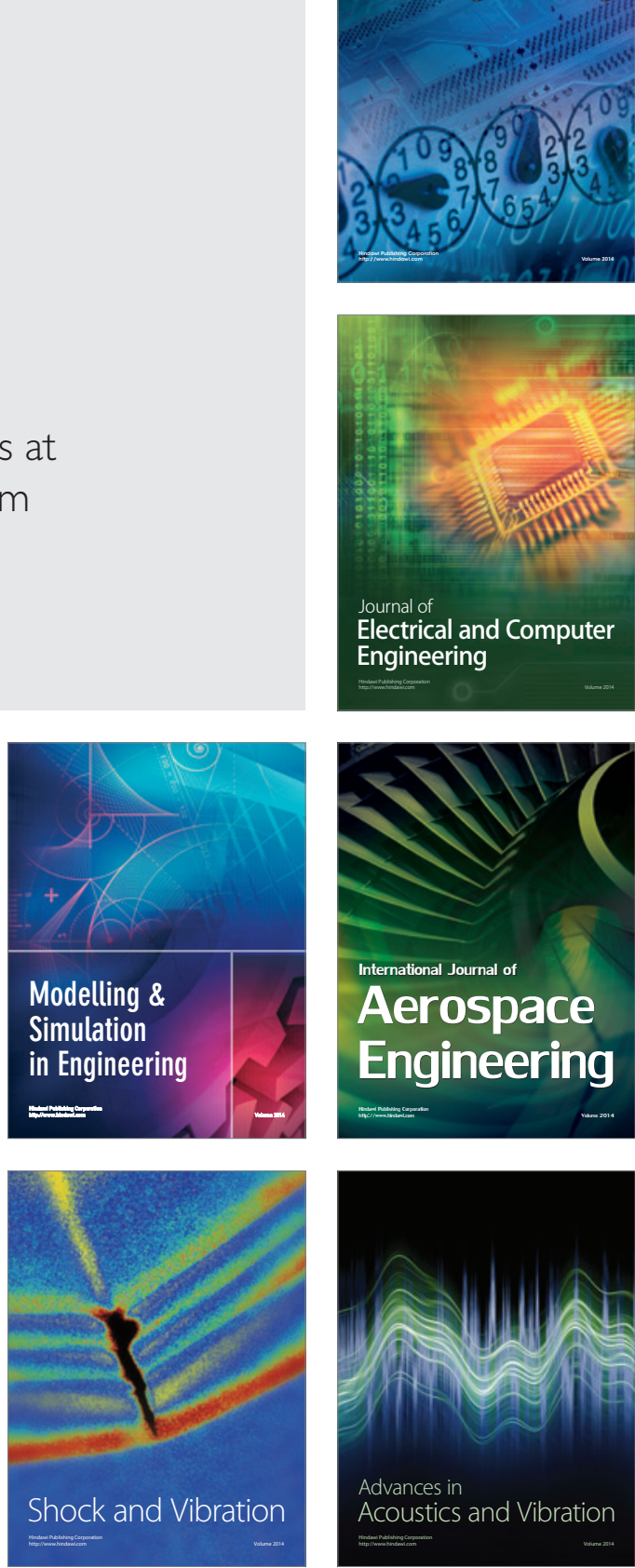